\title{
ESTIMATES AND ASYMPTOTIC EXPANSIONS FOR CONDENSER $P$-CAPACITIES. THE ANISOTROPIC CASE OF SEGMENTS.
}

\author{
ALAIN BONNAFÉ
}

\begin{abstract}
We provide estimates and asymptotic expansions of condenser $p$-capacities and focus on the anisotropic case of segments.

After preliminary results, we study $p$-capacities of points with respect to asymptotic approximations, positivity cases and convergence speed of descending continuity.

We introduce equidistant condensers to point out that the anisotropy caused by a segment in the $p$-Laplace equation is such that the Pólya-Szegö rearrangement inequality for Dirichlet type integrals yields a trivial lower bound. Moreover, when $p>N$, one cannot build an admissible solution for the segment, however small its length may be, by extending the case of a punctual obstacle.

Our main contribution is to provide a lower bound to the $N$-dimensional condenser $p$-capacity of a segment, by means of the $N$-dimensional and of the $(N-1)$-dimensional condenser $p$-capacities of a point. The positivity cases follow for $p$-capacities of segments. Our method could be extended to obstacles with codimensions $\geq 2$ in higher dimensions, such as surfaces in $\mathbb{R}^{4}$.

Introducing elliptical condensers, we obtain an estimate and the asymptotic expansion for the condenser 2-capacity of a segment in the plane. The topological gradient of the 2-capacity is not an appropriate tool to separate curves and obstacles with non-empty interior in 2D. In the case $p \neq 2$, elliptical condensers should prove useful to obtain further estimates of $p$-capacities of segments.
\end{abstract}

\section{INTRODUCTION}

1.1. Motivations and goals. This article deals with estimates of condenser $p$-capacities, $p \in(1, \infty)$, that is $p$-capacities of obstacles located within a bounded domain $\Omega \subset \mathbb{R}^{N}$. Our goal is to estimate such capacities when the interior of the obstacle is empty, and in particular when the codimension of the obstacle is $\geq 2$, as in the case of points in $\mathbb{R}^{2}$ and of segments in $\mathbb{R}^{3}$.

The literature often considers sets with null variational capacity, sometimes called 'polar sets' (21], $\S 2.1 .2)$, as under relevant assumptions, they are removable singularities for quasilinear elliptic partial differential equations ([30, 31] or [21], Thm. 2.116 and 3.15).

By contrast, the purpose of this article is to study estimates of positive $p$-capacities. Although estimating the capacity of a segment may seem to be a simple task at first glance, such an obstacle causes a strongly anisotropic effect in the $p$-Laplace problem, which has not been studied yet.

On the applicative side, the ability of estimating positive $p$-capacities of points in $2 \mathrm{D}$ and of segments in 3D may prove useful in the field of imaging, in particular by means of topological asymptotic methods. Topological asymptotic expansions (e.g. [29, 22, 23, 32, 6, 8, 3, 19]) assess the sensitivity of an appropriately chosen functional $J$, applied to the solution $u_{\varepsilon}$ of a partial differential equation in a domain $\Omega$, when the latter equation is perturbed in a compact $K_{\varepsilon}$ which geometric parameter $\varepsilon$ goes down to zero. They come in the form

$$
J\left(u_{\varepsilon}\right)=J_{0}\left(u_{0}\right)+\rho(\varepsilon) g\left(x_{0}\right)+o(\rho(\varepsilon)), \quad \forall \varepsilon \geq 0 \text { small enough, }
$$

where $\rho$ is a nonnegative function such that $\lim _{\varepsilon \rightarrow 0} \rho(\varepsilon)=0$ and where the scalar $g\left(x_{0}\right)$ is called the topological gradient at point $x_{0}$.

They were extensively studied for the Laplace equation [15, 2, 24 and for the Helmholtz equation [28. They are applied to perform various detection tasks in imaging (e.g. [5, 6, 7, 8, 19]).

As a smooth curve can be locally approximated by a segment of length small enough, the task of detecting a segment in a 2D image by a Laplace equation with Neumann boundary condition was

Date: March 27, 2013.

2010 Mathematics Subject Classification. 31C15, 31C45, 35J92, 41A60, $42 \mathrm{~B} 37$.

Key words and phrases. Capacity; Condenser p-capacity; Equidistant condenser; Elliptical condenser; $p$-Laplace equation; Nonlinear potential theory; Asymptotic analysis; Topological gradient; Topological sensitivity. 
studied in 11. But to perfom the task of detecting points in 2D and segments in 3D, one must turn to $p$-capacities, with $p>2$.

So as to be consistent with topological asymptotic expansions as defined in (1.1), we study a type of capacity which considers a set $K$ within a given bounded domain $\Omega$. Namely condenser p-capacities will be considered all through this article.

1.2. State of the art. Most available estimates of capacities deal with the harmonic or electrostatic case, that is $p=2$, either referring to variational capacities (e.g. [13, 33]) or to condenser capacities (e.g. [25, 26]).

Results usually come in the form of inequalities. Equalities are exceptions. When $p \neq 2$ actually, as mentioned by [16] $\S 2.11$, only were calculated $p$-capacities of spherical condensers.

One a priori expects the condenser $p$-capacity of compact $K$ in $\Omega$ to depend on the shape of $K$ but also on its localization within $\Omega$ and on the shape and size of $\Omega$. While the condenser capacity of a point $\left\{x_{0}\right\}$ may be approximated by condenser capacities of spherical compacts $\bar{B}\left(x_{0}, \varepsilon\right), \varepsilon \rightarrow 0$, the estimation of condenser capacities of segments remains an opened question. Trying to apply the descending continuity property of capacities, one may estimate capacities of ellipsoids. Some results are available for ellipsoids (e.g. [25, 33, 13]) but only in the harmonic case $p=2$. As for $p$-Laplace problems, most available results address the case of isolated singularities and of radial solutions ([17, 37]). Anisotropic solutions of the form $u(x)=|x|^{\lambda} \omega(x /|x|)$, where $\lambda \in \mathbb{R}$ and $\omega$ is defined on the unit sphere $S^{N-1}$, were studied for quasilinear equations with Dirichlet conditions in domains with conical boundary points ([34, 27]). To our best knowledge, the anisotropic effect caused by a segment in the $p$-Laplace equation has not been studied yet.

Lastly, with respect to positivity versus nullity cases of condenser $p$-capacities, clearly if the variational $p$-capacity of a compact $K$ in $\mathbb{R}^{N}$ is positive, then the condenser $p$-capacity of $K$ in a bounded domain is positive. The necessary and sufficient condition of nullity for the variational $p$-capacity of a set is well-known (see e.g. 21, §2.1.7 and [4, Thm. 4.1.2), i.e. parameter $p$ has to be less or equal the codimension of the set. But available results ([16], Theorems 2.26 and 2.27) about cases of nullity for condenser $p$-capacities only apply when the condenser $p$-capacity of a compact $K$ is null in all bounded domain $\Omega, K \subset \Omega \subset \mathbb{R}^{N}$.

1.3. Overview of article. Section 2 is devoted to definitions and preliminary estimation tools. To estimate the capacity of an obstacle with empty interior, due to the descending continuity property of capacities, it matters to estimate condenser $p$-capacities of decreasing sequences of obstacles $K_{\varepsilon}$ with non-empty interior, such that $\bigcap_{\varepsilon>0} K_{\varepsilon}$ reduces to the targeted obstacle. We show that one can calculate a condenser $p$-capacity by solving a $p$-Laplace equation with Dirichlet boundary condition, when the boundaries of the condenser are smooth. Then we give estimates for condenser $p$-capacities when the obstacle $K$ has a non-empty interior.

In section 3 , we apply the previous results to obtain asymptotic approximations and cases of positivity for condenser $p$-capacities of points. We study the convergence speed of descending continuity.

Our main contributions deal with condenser $p$-capacities of segments in section 4 . For this purpose, we first introduce equidistant condensers. As a first illustration of the strong anisotropy of the problem, we show that the Pólya-Szegö rearrangement inequality for Dirichlet type integrals fails to provide a valuable lower bound to the $p$-capacity of a segment. As a second illustration, when $p>N$, we show that one cannot simply derive an admissible solution for the segment $S_{\varepsilon}$, however small its length $\varepsilon>0$ may be, from the case of a punctual obstacle.

Taking into account the outcome of the previous experiments, our main contribution is to provide a lower bound to the condenser $p$-capacity of a segment $S$ in a $N$-dimensional bounded domain $\Omega$, by means of the $N$-dimensional $p$-capacity of a point and more importantly by means of the $(N-1)$-dimensional $p$-capacity of a point. We can then obtain directly positivity cases for condenser $p$-capacities of segments in an $N$-dimensional bounded domain $\Omega$. This method could be extended to obstacles of higher dimensions, e.g. for plane rectangles in $\mathbb{R}^{N}, N \geq 4$.

In section 4.6 we introduce elliptical condensers, defined in elliptic coordinates. The angular coordinate $\nu$ so to speak makes the dimension in which operates the $p$-Laplace equation, continuously change from $N$ for $\nu=0$ to $(N-1)$ for $\nu=\pi / 2$ and then back to $N$ for $\nu=\pi$. We provide an estimate for the condenser 2-capacity of a segment in the plane and its asymptotic expansion when 
the segment length goes down to 0 (in higher dimensions, this 2-capacity is null). In terms of topological asymptotic expansions, this result shows that the 2-condenser capacity in the plane is unable to separate curves and balls. When $p \neq 2$, elliptical condensers could prove useful to obtain further estimations of condenser $p$-capacities of segments.

For reader's convenience, two proofs requiring longer calculations are postponed to section 5 .

In all this article, let $p \in(1,+\infty)$ and $N \in \mathbb{N}, N \geq 1$. Let a bounded domain (open connected set) $\Omega \subset \mathbb{R}^{N}$ and a compact subset $K \subset \Omega$. The symbol $|E|$ denotes either the usual euclidean norm in $\mathbb{R}^{N}$ when $E \in \mathbb{R}^{N}$ or the $N$-dimensional Lebesgue measure when $E \subset \mathbb{R}^{N}$. $S^{N-1}$ will be the unit sphere in $\mathbb{R}^{N}$ and $A^{N-1}$ its surface area. For simplicity, denote $\beta:=(p-N) /(p-1) \in(-\infty, 1]$. It is convenient to remember that $p>N \Leftrightarrow \beta>0$ and that $\beta<1$, for all $N \geq 2$.

\section{Preliminary Results For CONDENSER CAPACITIES}

2.1. Definition of condenser $p$-capacities and basic properties. Since a Poincaré inequality holds in $W_{0}^{1, p}(\Omega)([4, \S 5.3)$, Heinonen et al. [16], Chap. 2, set

Definition 2.1. Let $W(K, \Omega):=\left\{v \in C_{0}^{\infty}(\Omega): v \geq 1\right.$ in $\left.K\right\}$. One defines

$$
C_{p, N}(K, \Omega):=\inf _{v \in W(K, \Omega)} \int_{\Omega}|\nabla v|^{p} .
$$

The nonnegative number $C_{p, N}(K, \Omega)$ is called the $p$-capacity of the condenser $(K, \Omega)$ or the condenser p-capacity of the obstacle $K$ in the bounded domain $\Omega$.

Using an approximation argument, one can prove that the set $W(K, \Omega)$ can be replaced in Definition 2.1 by the larger set

that is

$$
W_{0}(K, \Omega):=\left\{v \in W_{0}^{1, p}(\Omega) \cap C(\Omega): v \geq 1 \text { in } K\right\}
$$

$$
C_{p, N}(K, \Omega)=\inf _{v \in W_{0}(K, \Omega)} \int_{\Omega}|\nabla v|^{p}
$$

A function $v \in W_{0}(K, \Omega)$ is called an admissible function for the condenser.

Definition 2.1 can be extended to any subset $E \subset \Omega$, but we only need the case $E=K$ compact for estimation purposes in this article.

For simplicity, we henceforth drop the word 'condenser' and simply say ' $p$-capacity' instead of 'condenser $p$-capacity' when no confusion is possible. Similarly we drop the ' $N$ ' of ' $C_{p, N}(K, \Omega)$ ' simply writing ' $C_{p}(K, \Omega)$ ' whenever no confusion is possible about the dimension of the ambient space.

Condenser capacities comply with Choquet's axiomatic definition [11] of capacities. Three properties will be needed. After [16] $\$ 2.2$ it holds

Theorem 2.2. The set function $K \rightarrow C_{p}(K, \Omega)$, where $K$ is a compact included in the domain $\Omega \subset \mathbb{R}^{N}$, enjoys the following properties:

(i) (Monotony) If $K_{1} \subset K_{2} \subset \Omega$ then $C_{p}\left(K_{1}, \Omega\right) \leq C_{p}\left(K_{2}, \Omega\right)$.

(ii) (Monotony) If $K \subset \Omega_{1} \subset \Omega_{2}$ then $C_{p}\left(K, \Omega_{2}\right) \leq C_{p}\left(K, \Omega_{1}\right)$.

(iii) (Descending continuity) If $\left(K_{n}\right)_{n \geq 0}$ is a decreasing sequence of compact subsets of $\Omega$, that is

$$
\begin{gathered}
\Omega \supset K_{0} \supset K_{1} \supset \cdots \supset K_{n} \supset K_{n+1} \supset \cdots \text { and } K:=\bigcap_{n \geq 0} K_{n} \text {, then } \\
C_{p}(K, \Omega)=\lim _{n \rightarrow+\infty} C_{p}\left(K_{n}, \Omega\right) .
\end{gathered}
$$

2.2. Estimations of condenser $p$-capacity by means of a $p$-Laplace problem. Assume that both $\Omega$ and $K$ have smooth $C^{1}$-boundaries. Consider the following $p$-Laplace problem in $\Omega \backslash K$ with Dirichlet boundary condition:

$$
\begin{cases}-\Delta_{p}(u)=0 & \text { in } \Omega \backslash K \\ u=1 & \text { on } \partial K \\ u=0 & \text { on } \partial \Omega\end{cases}
$$

where $\Delta_{p}$ denotes the $p$-Laplace operator $\Delta_{p}(u):=\operatorname{div}\left(|\nabla u|^{p-2} \nabla u\right)$. 
We recall some well-known facts about Problem (2.3) (see e.g. [20, Thm. 2.16 or [4, $\$ 6.6$ for existence and unicity; [35, 38, 14, 21] for regularity properties; and [20, 14, 21] for the Maximum Principle):

- Problem (2.3) admits a unique solution $u \in W^{1, p}(\Omega \backslash K)$.

- One can define solution $u$ as being the unique function that minimizes the Fréchet differentiable, strictly convex and coercive functional

$$
J: v \in W^{1, p}(\Omega \backslash K) \mapsto \int_{\Omega \backslash K}|\nabla v|^{p}
$$

in the affine space $g+W_{0}^{1, p}(\Omega \backslash K)$, where $g \in C_{0}^{\infty}(\Omega)$ is chosen such that $g=1$ on a neighborhood $\Omega^{\prime}$ of $K$, with $K \subset \subset \Omega^{\prime} \subset \subset \Omega$. In other words,

$$
\{u\}=\underset{v \in g+W_{0}^{1, p}(\Omega \backslash K)}{\operatorname{argmin}} \int_{\Omega \backslash K}|\nabla v|^{p} .
$$

- Function $u$ is continuous in $\overline{\Omega \backslash K}$ (after a redefinition in a set of zero measure) and $u$ is $C^{1}$ in $\Omega \backslash K$. In particular it holds $u=0$ on $\partial \Omega$ and $u=1$ on $\partial K$ pointwise.

- It holds

$$
0<u(x)<1, \quad \forall x \in \Omega \backslash K
$$

Proposition 2.3. Let $K$ be a compact set of a bounded domain $\Omega \subset \mathbb{R}^{N}$, both with $C^{1}$-boundaries. Let $u \in W^{1, p}(\Omega \backslash K) \cap C(\overline{\Omega \backslash K})$ be the unique solution to Problem 2.3. . Then

$$
C_{p}(K, \Omega)=\int_{\Omega \backslash K}|\nabla u|^{p} .
$$

Moreover let $\tilde{u}$ be the extension of $u$ in $\Omega$ obtained by setting $\tilde{u}=1$ in $K$. Then $\tilde{u} \in W_{0}(K, \Omega)$ and $\tilde{u}$ minimizes Problem (2.2), that is

$$
\{\tilde{u}\}=\underset{v \in W_{0}(K, \Omega)}{\operatorname{argmin}} \int_{\Omega}|\nabla v|^{p}
$$

and

$$
C_{p}(K, \Omega)=\int_{\Omega}|\nabla \tilde{u}|^{p}
$$

Proof. The regularity of function $u$ entails that $\tilde{u} \in W_{0}^{1, p}(\Omega) \cap C(\bar{\Omega})$. As $\tilde{u}=1$ in $K$, it follows that $\tilde{u} \in W_{0}(K, \Omega)$. Obviously $\nabla \tilde{u}=0$ in $\stackrel{\circ}{K}$. Hence according to 2.2

$$
C_{p}(K, \Omega) \leq \int_{\Omega}|\nabla \tilde{u}|^{p}=\int_{\Omega \backslash K}|\nabla u|^{p} .
$$

Conversely, according to definition (2.1), let $\left(u_{n}\right)_{n \geq 0}$ a sequence $\subset W(K, \Omega)$ such that

$$
C_{p}(K, \Omega)=\lim _{n \rightarrow+\infty} \int_{\Omega}\left|\nabla u_{n}\right|^{p} .
$$

For all $n \geq 0$, define $w_{n}:=\inf \left(u_{n}, 1\right)$ in $\Omega$. It follows from [16] Thm 1.20 that

$$
w_{n} \in W_{0}^{1, p}(\Omega) \cap C(\Omega)
$$

and that

$$
\left|\nabla w_{n}(x)\right| \leq\left|\nabla u_{n}(x)\right|, \quad \text { for a.e. } x \in \Omega \text {. }
$$

In addition the obstacle condition $u_{n} \geq 1$ in $K$ implies that $w_{n}=1$ in $K$ and $\nabla w_{n}=0$ in $\stackrel{\circ}{K}$. Hence $w_{n} \in W_{0}(K, \Omega)$ with

$$
\int_{\Omega}\left|\nabla w_{n}\right|^{p} \leq \int_{\Omega}\left|\nabla u_{n}\right|^{p}
$$

Let $v_{n}$ be the restriction of $w_{n}$ to $\Omega \backslash K$. We check that $v_{n}-g \in W_{0}^{1, p}(\Omega \backslash K)$ where function $g$ was defined in 2.5. Therefore according to 2.5 for all $n \geq 0$ it holds:

$$
J(u)=\int_{\Omega \backslash K}|\nabla u|^{p} \leq \int_{\Omega \backslash K}\left|\nabla v_{n}\right|^{p}=\int_{\Omega}\left|\nabla w_{n}\right|^{p} \leq \int_{\Omega}\left|\nabla u_{n}\right|^{p} .
$$


Letting $n \rightarrow+\infty$ yields

$$
\int_{\Omega \backslash K}|\nabla u|^{p} \leq C_{p}(K, \Omega)
$$

Comparing with 2.9 one concludes

$$
C_{p}(K, \Omega)=\int_{\Omega}|\nabla \tilde{u}|^{p}=\int_{\Omega \backslash K}|\nabla u|^{p} .
$$

Equality (2.7) is thus proved. As we already noticed that $\tilde{u} \in W_{0}(K, \Omega)$, it also follows that

$$
\{\tilde{u}\}=\underset{v \in W_{0}(K, \Omega)}{\operatorname{argmin}}\left\{\int_{\Omega}|\nabla v|^{p}\right\}
$$

which completes the proof.

Note that after (2.6), obviously

$$
0<\tilde{u}(x)<1, \quad \forall x \in \Omega \backslash K .
$$

Thus after Proposition 2.3 one can estimate the capacity of the condenser $(K, \Omega)$ by estimating the energy of the solution to Problem $(2.3)$ when boundaries $\partial K$ and $\partial \Omega$ are smooth enough.

If boundaries $\partial K$ or $\partial K$ are not $C^{1}$, then thanks to the two monotony properties $(i)$ and $(i i)$ of Theorem 2.2 one can estimate $C_{p}(K, \Omega)$ as long as $K$ (resp. $\Omega$ ) can be properly approximated respectively by (a sequence of) some other compact sets (resp. open sets) with $C^{1}$-boundaries to which one may in turn apply Proposition 2.3. This approximation technique will be applied in subsection 2.4 hereafter.

It is convenient to extend the definition of 'admissible function' for a condenser, given in Definition 2.1 as follows : let $v \in W^{1, p}(\Omega \backslash K) \cap C(\overline{\Omega \backslash K})$ such that $v=0$ on $\partial \Omega$ and $v=1$ on $\partial K$. Let $\tilde{v}$ be the extension of $v$ in $\Omega$ obtained by setting $\tilde{v}=1$ in $K$. Clearly $\tilde{v}$ is admissible for the condenser $(K, \Omega)$ in the sense of Definition 2.1. By extension we thus say that function $v$ is admissible for the condenser $(K, \Omega)$.

2.3. Asymptotic expansions of capacity for spherical condensers. Let a point $x_{0} \in \mathbb{R}^{N}$, two numbers $0<\varepsilon<R$ and the concentric balls $\bar{B}_{\varepsilon}:=\bar{B}\left(x_{0}, \varepsilon\right)$ and $B_{R}:=B\left(x_{0}, R\right)$. For simplicity, we denote $C_{p}(\varepsilon, R)$ the $p$-capacity of the spherical condenser $\left(\bar{B}\left(x_{0}, \varepsilon\right), B\left(x_{0}, R\right)\right)$. We recall and detail hereafter the well-known result (e.g. [16], §2.11) about the spherical condenser $\left(\bar{B}_{\varepsilon}, B_{R}\right)$.

Proposition 2.4. Denote $s_{p, N} \in W^{1, p}\left(B_{R} \backslash \bar{B}_{\varepsilon}\right)$ the unique solution to Problem (2.3) when $K=\bar{B}_{\varepsilon}$ and $\Omega=B_{R}$. Denote $r=\left|x-x_{0}\right|$ for all $x \in B_{R} \backslash \bar{B}_{\varepsilon}$.

(1) If $p=N$, then for all $x \in B_{R} \backslash \bar{B}_{\varepsilon}$ it holds:

$$
\begin{gathered}
\left\{\begin{array}{l}
s_{p, N}(x)=[\log (R / r) / \log (R / \varepsilon)], \\
\left|\nabla s_{p, N}(x)\right|=[r \log (R / \varepsilon)]^{-1},
\end{array}\right. \\
C_{p}(\varepsilon, R)=A^{N-1}[\log (R / \varepsilon)]^{1-p},
\end{gathered}
$$

and for $\varepsilon>0$ small enough:

$$
C_{p}(\varepsilon, R)=A^{N-1}[-\log \varepsilon]^{1-p}[1+(p-1)(\log R / \log \varepsilon)+o(1 / \log \varepsilon)] .
$$

(2) If $p \neq N$, then for all $x \in B_{R} \backslash \bar{B}_{\varepsilon}$ it holds:

$$
\begin{aligned}
& \qquad\left\{\begin{array}{l}
s_{p, N}(x)=\left(R^{\beta}-r^{\beta}\right) /\left(R^{\beta}-\varepsilon^{\beta}\right), \\
\left|\nabla s_{p, N}(x)\right|=\left|\frac{\beta}{R^{\beta}-\varepsilon^{\beta}}\right| r^{\beta-1},
\end{array}\right. \\
& \qquad C_{p}(\varepsilon, R)=A^{N-1}|\beta|^{p-1}\left|R^{\beta}-\varepsilon^{\beta}\right|^{1-p}, \\
& \text { and for } \varepsilon>0 \text { small enough } \\
& \left\{\begin{array}{l}
C_{p}(\varepsilon, R)=A^{N-1} \beta^{p-1} R^{N-p}\left[1+(p-1)(\varepsilon / R)^{\beta}+o\left(\varepsilon^{\beta}\right)\right], \\
C_{p}(\varepsilon, R)=A^{N-1}(-\beta)^{p-1} \varepsilon^{N-p}\left[1+(p-1)(\varepsilon / R)^{-\beta}+o\left(\varepsilon^{-\beta}\right)\right],
\end{array} \quad \text { if } p<N .\right.
\end{aligned}
$$

A detailed proof is made available in subsection 5.1 on page 18 It is obtained solving Problem 2.3 in spherical coordinates and then applying Proposition 2.3 . 


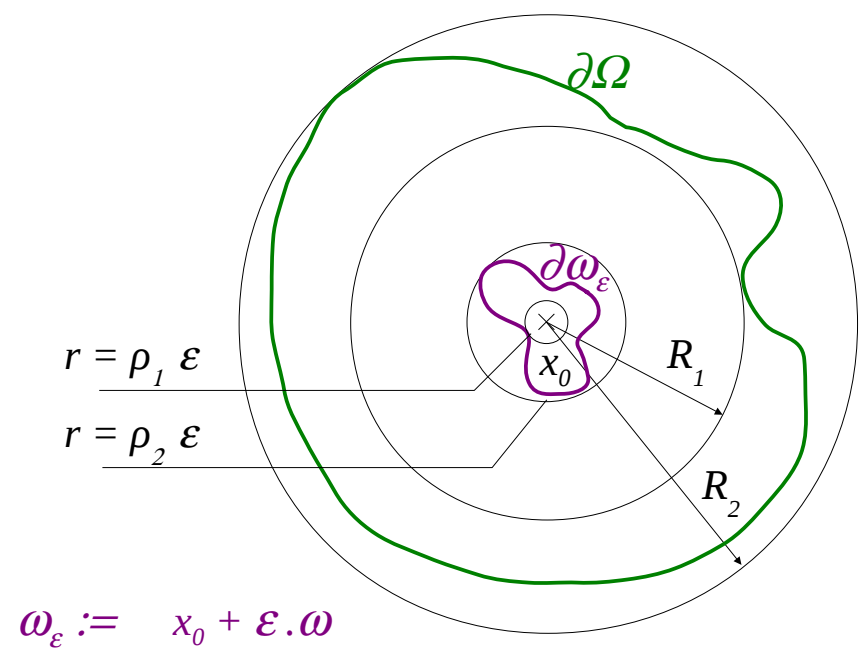

FIGURE 1. A condenser which obstacle $\bar{\omega}_{\varepsilon}$ has a non-empty interior.

2.4. Capacities of condensers which obstacle has non-empty interior. Let a point $x_{0} \in \Omega$. Let the two numbers

$$
R_{1}:=\sup \left\{R>0 ; B\left(x_{0}, R\right) \subset \Omega\right\}>0 \quad \text { and } \quad R_{2}:=\inf \left\{R>0 ; \Omega \subset B\left(x_{0}, R\right)\right\} .
$$

Let a non-empty bounded domain $\omega \subset \mathbb{R}^{N}$ such that $0 \in \omega$. Let the two numbers

$$
\rho_{1}:=\sup \left\{\rho>0 ; B\left(x_{0}, \rho\right) \subset \omega\right\} \quad \text { and } \quad \rho_{2}:=\inf \left\{\rho>0 ; \omega \subset B\left(x_{0}, \rho\right)\right\} \text {. }
$$

Let $\omega_{\varepsilon}:=x_{0}+\varepsilon \cdot \omega \subset B\left(x_{0}, R_{1}\right)$ for $\varepsilon>0$ small enough and consider the condenser $\left(\bar{\omega}_{\varepsilon}, \Omega\right)$ as shown on figure 1

Proposition 2.5. The following asymptotic inequalities hold.

(1) If $p=N$, then:

$$
\begin{gathered}
-A^{N-1}(p-1) \log \left(R_{2} / \rho_{1}\right)[-\log \varepsilon]^{-p}+o\left([\log \varepsilon]^{-p}\right) \\
\leq C_{p}\left(\bar{\omega}_{\varepsilon}, \Omega\right)-A^{N-1}[-\log \varepsilon]^{1-p} \leq \\
-A^{N-1}(p-1) \log \left(R_{1} / \rho_{2}\right)[-\log \varepsilon]^{-p}+o\left([\log \varepsilon]^{-p}\right) .
\end{gathered}
$$

(2) If $p>N$, then:

$$
\begin{gathered}
A^{N-1} \beta^{p-1} R_{2}^{N-p}\left[1+(p-1)\left(\rho_{1} \varepsilon / R_{2}\right)^{\beta}+o\left(\varepsilon^{\beta}\right)\right] \\
\leq C_{p}\left(\bar{\omega}_{\varepsilon}, \Omega\right) \leq \\
A^{N-1} \beta^{p-1} R_{1}^{N-p}\left[1+(p-1)\left(\rho_{2} \varepsilon / R_{1}\right)^{\beta}+o\left(\varepsilon^{\beta}\right)\right] .
\end{gathered}
$$

(3) If $p<N$, then:

$$
\begin{gathered}
A^{N-1}(-\beta)^{p-1}\left(\rho_{1} \varepsilon\right)^{N-p}\left[1+(p-1)\left(\rho_{1} \varepsilon / R_{2}\right)^{-\beta}+o\left(\varepsilon^{-\beta}\right)\right] \\
\leq C_{p}\left(\bar{\omega}_{\varepsilon}, \Omega\right) \leq \\
A^{N-1}(-\beta)^{p-1}\left(\rho_{2} \varepsilon\right)^{N-p}\left[1+(p-1)\left(\rho_{2} \varepsilon / R_{1}\right)^{-\beta}+o\left(\varepsilon^{-\beta}\right)\right] .
\end{gathered}
$$


Proof. Let four positive real numbers $\rho^{\prime}, \rho^{\prime \prime}, R^{\prime}$ and $R^{\prime \prime}$ such that

$$
B\left(x_{0}, \rho^{\prime} \varepsilon\right) \subset \omega_{\varepsilon} \subset B\left(x_{0}, \rho^{\prime \prime} \varepsilon\right) \subset B\left(x_{0}, R^{\prime}\right) \subset \Omega \subset B\left(x_{0}, R^{\prime \prime}\right) .
$$

According to monotony properties $(i)$ and $(i i)$ of Theorem 2.2 the following inequalities hold

$$
C_{p}\left(\rho^{\prime} \varepsilon, R^{\prime \prime}\right) \leq C_{p}\left(\bar{B}_{\rho^{\prime} \varepsilon}, \Omega\right) \leq C_{p}\left(\bar{\omega}_{\varepsilon}, \Omega\right) \leq C_{p}\left(\bar{B}_{\rho^{\prime \prime} \varepsilon}, \Omega\right) \leq C_{p}\left(\rho^{\prime \prime} \varepsilon, R^{\prime}\right) .
$$

The formula of $C_{p}(\rho, R)$ provided by Theorem 2.4 shows that the map $(\rho, R) \in \mathbb{R}^{2} \mapsto C_{p}(\rho, R)$ is continuous in $\left\{(\rho, R) \in \mathbb{R}^{2} \mid 0<\rho<R\right\}$.

Hence letting $R^{\prime} \nearrow R_{1}, R^{\prime \prime} \searrow R_{2}, \rho^{\prime} \nearrow \rho_{1}$ and $\rho^{\prime \prime} \searrow \rho_{2}$ in inequalities (2.13), it follows that

$$
C_{p}\left(\rho_{1} \varepsilon, R_{2}\right) \leq C_{p}\left(\bar{\omega}_{\varepsilon}, \Omega\right) \leq C_{p}\left(\rho_{2} \varepsilon, R_{1}\right)
$$

Then consider the asymptotic expansions provided by Theorem 2.4 for the lower-bound $C_{p}\left(\rho_{1} \varepsilon, R_{2}\right)$ and for the upper-bound $C_{p}\left(\rho_{2} \varepsilon, R_{1}\right)$. The expansions claimed in Proposition 2.5 straighforwardly follow, whether $p=N, p>N$ or $p<N$.

Note that no assumption is required about the smoothness of boundaries $\partial \omega$ and $\partial \Omega$.

Remark 2.6. The expansions stated in Proposition 2.5 are actually topological asymptotic expansions in the sense of 1.1

(1) If $p=N$, then the expansion reads

$$
C_{p}\left(\bar{\omega}_{\varepsilon}, \Omega\right)=A^{N-1}[-\log \varepsilon]^{1-p}+o\left([-\log \varepsilon]^{1-p}\right) .
$$

The topological gradient equals $A^{N-1}$. It is constant in $\Omega$. It does not depend on the shape of the compact $\bar{\omega}$ nor on that of the domain $\Omega$.

(2) If $p<N$ and if $\omega$ is the unit ball, then the expansion reads

$$
C_{p}\left(\bar{B}_{\varepsilon}, \Omega\right)=A^{N-1}(-\beta)^{p-1} \varepsilon^{N-p}+o\left(\varepsilon^{N-p}\right) .
$$

The topological gradient equals $A^{N-1}(-\beta)^{p-1}$. It is constant in $\Omega$. It does not depend on the shape of the domain $\Omega$.

(3) In the harmonic case $p=2$ and for $N=2$ or $N=3$, the results hereabove comply with the topological expansion previously proved in [15] for the Laplace equation with Dirichlet boundary condition.

Remark 2.7. According to expansions stated in Proposition 2.5, the domain $\Omega$, through parameters $R_{1}$ and $R_{2}$, does not impact the main term of the asymptotic expansion of the capacity $C_{p}\left(\bar{\omega}_{\varepsilon}, \Omega\right)$ when $p \leq N$.

In contrast when $p>N$, the localization of $x_{0}$ within $\Omega$ and the shape of $\Omega$ determine the main term of the expansion through parameters $R_{1}$ and $R_{2}$. This case exemplifies a major difference between the concept of condenser capacities in a bounded domain $\Omega$ and that of variational capacities in $\mathbb{R}^{N}$.

\section{Condenser $p$-CAPACITY OF A POINT AND APPROXimations}

\subsection{Condenser $p$-capacity of a point.}

Proposition 3.1. Let $x_{0}$ be a point of a bounded domain $\Omega \subset \mathbb{R}^{N}$. The following positivity rule holds:

$$
C_{p}\left(\left\{x_{0}\right\}, \Omega\right)>0 \quad \text { if and only if } p>N \text {. }
$$

Moreover, if $p>N$, then:

$$
A^{N-1} \beta^{p-1} R_{2}^{N-p} \leq C_{p}\left(\left\{x_{0}\right\}, \Omega\right) \leq A^{N-1} \beta^{p-1} R_{1}^{N-p}
$$

where $R_{1}:=\sup \left\{R>0 ; B\left(x_{0}, R\right) \subset \Omega\right\}$ and $R_{2}:=\inf \left\{R>0 ; \Omega \subset B\left(x_{0}, R\right)\right\}$.

In particular, if $p>N$ and if $\Omega=B\left(x_{0}, R\right)$, then it holds

$$
C_{p}\left(\left\{x_{0}\right\}, B_{R}\right)=A^{N-1} \beta^{p-1} R^{N-p} .
$$


Proof. As $x_{0} \in \Omega$, there exists $\delta>0$ such that $B\left(x_{0}, \delta\right) \subset \Omega$. Let $\omega:=B(0, \delta)$ and $\omega_{\varepsilon}:=x_{0}+\varepsilon \omega$ for all $\varepsilon>0$.

For all $\varepsilon \in(0,1)$, the estimates of $C_{p}\left(\bar{\omega}_{\varepsilon}, \Omega\right)$ stated in Proposition 2.5 hold. As

$$
\left\{x_{0}\right\}=\bigcap_{\varepsilon \in(0,1)} \bar{\omega}_{\varepsilon},
$$

it follows from the descending continuity property of Theorem 2.2 that

$$
\lim _{\varepsilon \rightarrow 0} C_{p}\left(\bar{\omega}_{\varepsilon}, \Omega\right)=C_{p}\left(\left\{x_{0}\right\}, \Omega\right) .
$$

Passing to the limit when $\varepsilon \rightarrow 0$ in estimates of $C_{p}\left(\bar{\omega}_{\varepsilon}, \Omega\right)$ provided by Proposition 2.5 the claimed results follow whether $p=N, p>N$ or $p<N$.

3.2. Speed of convergence of descending continuity. According to the descending property of Theorem 2.2. one can approximate the capacity of an obstable with zero measure by calculating capacities of obstacles with positive measures going down to zero. From this perspective, the speed of convergence of descending continuity becomes a point of interest.

This question can be answered in the case of a point. If one wishes to obtain an estimate of the capacity of a point, one can calculate the capacity of a ball with a small enough $r$. How small should this radius be, depending on the maximum acceptable error for the value of the capacity of the point?

Proposition 3.2. If $p>N$, for $0<r<R$, it holds

$$
C_{p}\left(\bar{B}\left(x_{0}, r\right), B\left(x_{0}, R\right)\right)-C_{p}\left(\left\{x_{0}\right\}, B\left(x_{0}, R\right)\right)=O\left(r^{\beta}\right)
$$

Proof. The claimed estimate follows straightforwardly from the expansion stated in Proposition 2.4 in the case $p>N$ and from the value of $C_{p}\left(\left\{x_{0}\right\}, B\left(x_{0}, R\right)\right)$ provided by Proposition 3.1.

When $p>N \geq 2$, it holds $0<\beta<1$. The speed of convergence to zero, that is $O\left(r^{\beta}\right)$, is slow when $r \rightarrow 0$. Moreover according to Proposition 2.4, for all $\varepsilon \in(0, R)$ it holds

$$
C_{p}(\varepsilon, R)=A^{N-1} \beta^{p-1}\left(R^{\beta}-\varepsilon^{\beta}\right)^{1-p} .
$$

Hence

$$
\lim _{\varepsilon \rightarrow 0} \frac{\partial C_{p}(\varepsilon, R)}{\partial \varepsilon}=+\infty .
$$

\section{Estimates of $p$-CAPACities of Segments}

In this section, a segment will be a compact set $S_{\varepsilon} \subset \mathbb{R}^{N}, N \geq 2$, defined as follows

$$
S_{\varepsilon}:=\left\{x_{0}+z \tau ; z \in[-\varepsilon / 2, \varepsilon / 2]\right\}
$$

where $x_{0}$ is the center of the segment, $\varepsilon>0$ its length and $\tau \in \mathbb{R}^{N}$ is a unit vector.

4.1. Equidistant condensers. For all $x \in \mathbb{R}^{N}$ and all subset $E \subset \mathbb{R}^{N}$, we denote the distance $d(x, E)=\inf \left\{\left|x^{\prime}-x\right| ; x^{\prime} \in E\right\}$.

Definition 4.1. Let $0<\eta<R$. Let the compact $K_{\eta}:=\left\{x \in \mathbb{R}^{N} \mid d\left(x, S_{\varepsilon}\right) \leq \eta\right\}$ and the bounded domain $\Omega_{R}:=\left\{x \in \mathbb{R}^{N} \mid d\left(x, S_{\varepsilon}\right)<R\right\}$. We say that $\left(K_{\eta}, \Omega_{R}\right)$ is an equidistant condenser derived from the segment $S_{\varepsilon}$.

Some notations and remarks are useful.

(1) Let $z$ be an axis passing through the point $x_{0}$ and parallel to the segment $S_{\varepsilon}$. Due to the symmetry of revolution of the condenser $\left(K_{\eta}, \Omega_{R}\right)$ around the $z$-axis, it is convenient to use the cylindrical coordinates $x=(z, y)=(z, r, \xi)$, with $z \in \mathbb{R}, y=r \xi \in \mathbb{R}^{N-1}, r \geq 0$ and $\xi \in S^{N-2}$. Let $A$ (resp. $B$ ) the point of cylindrical coordinates $(z=-\varepsilon / 2, r=0$ ) (resp. $(z=\varepsilon / 2, r=0))$.

Let

$$
C:=\left\{x \in \Omega_{R} \backslash K_{\eta} ;|z|<\varepsilon / 2\right\}
$$

be the open cylindrical subset of $\Omega_{R} \backslash K_{\eta}$ and

$$
S_{ \pm}:=\left\{x \in \Omega_{R} \backslash K_{\eta} ; \pm z>\varepsilon / 2\right\}
$$

the two open half-spherical subsets of $\Omega_{R} \backslash K_{\eta}$.

Denote $S:=S_{-} \cup S_{+}$. In particular $\left(\Omega_{R} \backslash K_{\eta}\right) \backslash(C \cup S)$ is of zero Lebesgue measure. 


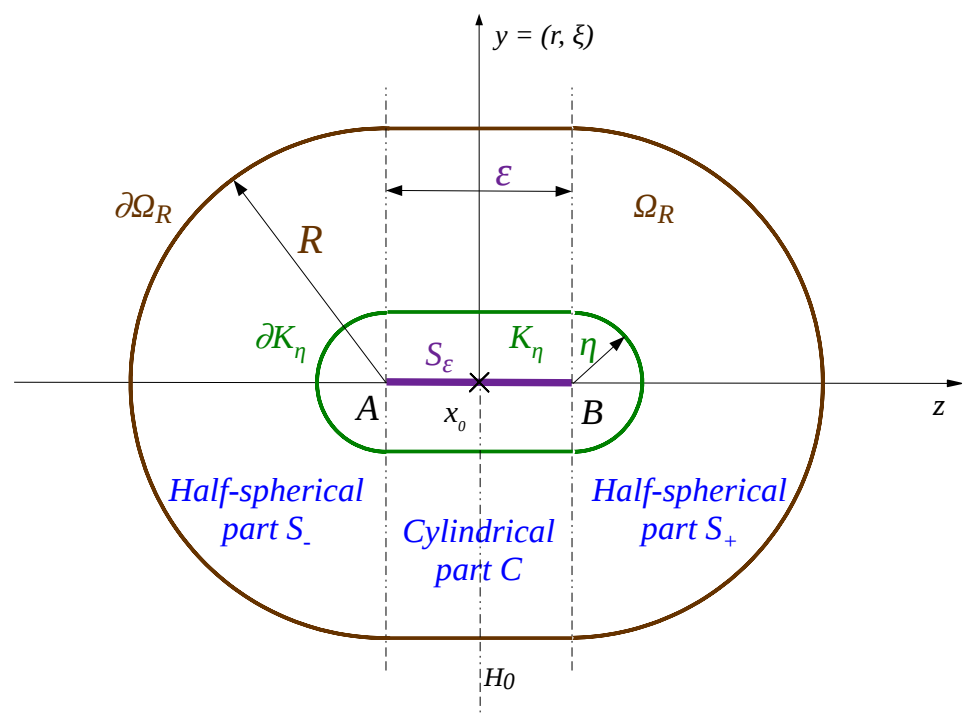

Figure 2. An equidistant condenser $\left(K_{\eta}, \Omega_{R}\right)$ derived from the segment $S_{\varepsilon}$.

(2) As in subsection 2.2 , we denote $u \in W^{1, p}\left(\Omega_{R} \backslash K_{\eta}\right) \cap C\left(\overline{\Omega_{R} \backslash K_{\eta}}\right) \cap C^{1}\left(\Omega_{R} \backslash K_{\eta}\right)$ the unique solution to Problem (2.3) when $K=K_{\eta}$ and $\Omega=\Omega_{R}$. According to Proposition 2.3 the $p$-capacity of the condenser $\left(K_{\eta}, \Omega_{R}\right)$ is given by

$$
C_{p, N}\left(K_{\eta}, \Omega_{R}\right)=\int_{C \cup S}|\nabla u|^{p} d x
$$

where $\nabla$ denotes the gradient operator in $\mathbb{R}^{N}$ and $d x$ the Lebesgue measure in $\mathbb{R}^{N}$.

Moreover $S_{\varepsilon}$ is invariant by the orthogonal symmetry $(z, y) \mapsto(-z, y)$ relative to

$H_{0}:=\{z=0\}$. Thus the condenser $\left(K_{\eta}, \Omega_{R}\right)$ enjoys the same symmetry and so does $u$ due to uniqueness of the solution to Problem 2.3 . .

(3) Denote $s_{p, N}$ the admissible function minimizing the energy of the $N$-dimensional spherical condenser $\left(\bar{B}\left(x_{0}, \eta\right), B\left(x_{0}, R\right)\right)$. After Proposition 2.3 it holds

$$
C_{p, N}(\eta, R)=\int_{B\left(x_{0}, R\right) \backslash B\left(x_{0}, \eta\right)}\left|\nabla s_{p, N}\right|^{p} d x .
$$

The values of $s_{p, N}$ and $C_{p, N}(\eta, R)$ were provided in Proposition 2.4

(4) For all $a \in[-\varepsilon / 2, \varepsilon / 2]$, let $H_{a}$ be the affine hyperplane $\{z=a\}$ and $\left\{x_{a}\right\}$ the intersection between $H_{a}$ and the $z$-axis.

It is pivotal to note that $\left(K_{\eta} \cap H_{a}, \Omega_{R} \cap H_{a}\right)$ is a $(N-1)$-dimensional spherical condenser. The admissible function minimizing the energy of this condenser is denoted $s_{p, N-1}$. Similarly after Proposition 2.3, it holds

$$
C_{p, N-1}(\eta, R)=\int_{B_{N-1}\left(x_{a}, R\right) \backslash B_{N-1}\left(x_{a}, \eta\right)}\left|\nabla_{y} s_{p, N-1}\right|^{p} d y,
$$

where $B_{N-1}$ denotes a $(N-1)$-dimensional ball, $\nabla_{y}$ is the gradient operator in $\mathbb{R}^{N-1}$ and $d y$ the Lebesgue measure in $\mathbb{R}^{N-1}$. The values of $s_{p, N-1}$ and $C_{p, N-1}(\eta, R)$ were provided in Proposition 2.4

4.2. Pólya-Szegö rearrangement inequality for Dirichlet type integrals. While the definition 2.1 of a condenser $p$-capacity allows to obtain upper bounds by calculating energies of admissible functions, obtaining a lower bound to a capacity is a more difficult task. In [25, 26] G. Pólya and G. Szegö showed in the harmonic case $p=2$, that the so called Schwarz symmetrization can provide a lower bound to a condenser 2-capacity. More recently Brothers and Ziemer [9, 12, 10] extended this 
method, known as the Pólya-Szegö rearrangement inequality for Dirichlet type integrals $\int_{\Omega}|\nabla u|^{p}$, for all $p \in[1, \infty)$.

So let us apply the Pólya-Szegö rearrangement inequality for Dirichlet type integrals, to obtain a lower-bound to the $p$-capacity of a segment $S_{\varepsilon}$.

- For $0<\eta<R$, let the equidistant condenser $\left(K_{\eta}, \Omega_{R}\right)$. According to definition 2.1 and Proposition 2.3. let

$$
u \in W^{1, p}\left(\Omega_{R} \backslash K_{\eta}\right) \cap C\left(\overline{\Omega_{R} \backslash K_{\eta}}\right) \cap C^{1}\left(\Omega_{R} \backslash K_{\eta}\right)
$$

the solution of Problem $(2.3)$. Let $\tilde{u} \in W_{0}^{1, p}\left(\Omega_{R}\right)$ the extension of $u$ obtained by setting $\tilde{u}=1$ in $K_{\eta}$. Recall

and

$$
C_{p}\left(K_{\eta}, \Omega_{R}\right)=\int_{\Omega_{R} \backslash K_{\eta}}|\nabla u|^{p}=\|\nabla \tilde{u}\|_{L^{p}\left(\Omega_{R}\right)}^{p}
$$

$$
0<\tilde{u}(x)<1, \quad \forall x \in \Omega_{R} \backslash K_{\eta} .
$$

- Let $\Omega^{\sharp}$ the ball of $\mathbb{R}^{N}$ centered at the origin 0 such that $\left|\Omega^{\sharp}\right|=\left|\Omega_{R}\right|$. The radius $R^{\sharp}$ of $\Omega^{\sharp}$ is given by

$$
R^{\sharp}:=\left[R^{N-1}\left(R+\frac{A^{N-2}}{A^{N-1}} \varepsilon\right)\right]^{\frac{1}{N}}
$$

and it holds $R^{\sharp}>R$.

Similarly we denote $K^{\sharp}$ the ball of $\mathbb{R}^{N}$ centered at the origin 0 such that $\left|K^{\sharp}\right|=\left|K_{\eta}\right|$. The radius $\eta^{\sharp}$ of $K^{\sharp}$ is given by

$$
\eta^{\sharp}:=\left[\eta^{N-1}\left(\eta+\frac{A^{N-2}}{A^{N-1}} \varepsilon\right)\right]^{\frac{1}{N}} .
$$

- Let $\mu_{\tilde{u}}$ the distribution function of $\tilde{u}$ defined by

$$
\mu_{\tilde{u}}(t):=\left|\left\{x \in \Omega_{R} ;|\tilde{u}(x)|>t\right\}\right|, \quad \forall t \geq 0 .
$$

It holds $\mu_{\tilde{u}}(0)=\left|\Omega_{R}\right|$ and $\lim _{t \rightarrow 1, t<1} \mu_{\tilde{u}}(t)=\left|K_{\eta}\right|$.

- Let $u^{*}$ the non-increasing rearrangement of $\tilde{u}$ defined by

$$
u^{*}(s):=\inf \left\{t \geq 0 ; \mu_{\tilde{u}}(t) \leq s\right\}, \quad \forall s \in\left(0,\left|\Omega_{R}\right|\right] .
$$

It holds $u^{*}\left(\left|K_{\eta}\right|\right)=1$ and $u^{*}\left(\left|\Omega_{R}\right|\right)=0$.

- Let $u^{\sharp}$ the symmetric rearrangement of $\tilde{u}$ defined by

$$
u^{\sharp}(x):=u^{*}\left(A^{N-1}|x|^{N}\right), \quad \forall x \in \Omega^{\sharp} .
$$

It holds

$$
u^{\sharp}(x)=1, \forall x \in \partial K_{\eta^{\sharp}} \text { and } u^{\sharp}(x)=0, \forall x \in \partial \Omega_{R^{\sharp}} .
$$

The Pólya-Szegö rearrangement inequality for Dirichlet type integrals reads

$$
\left\|\nabla u^{\sharp}\right\|_{L^{p}\left(\Omega^{\sharp}\right)}^{p} \leq\|\nabla \tilde{u}\|_{L^{p}\left(\Omega_{R}\right)}^{p} .
$$

As $u^{\sharp}$ is an admissible function for the spherical condenser $\left(K_{\eta^{\sharp}}, \Omega_{R^{\sharp}}\right)$, it follows

$$
C_{p}\left(K_{\eta^{\sharp}}, \Omega_{R^{\sharp}}\right) \leq\left\|\nabla u^{\sharp}\right\|_{L^{p}\left(\Omega^{\sharp}\right)}^{p} .
$$

Therefore

$$
C_{p}\left(K_{\eta^{\sharp}}, \Omega_{R^{\sharp}}\right) \leq C_{p}\left(K_{\eta}, \Omega_{R}\right) .
$$

- As $\lim _{\eta \rightarrow 0} \eta^{\sharp}=0$, it holds $\bigcap_{\eta>0} K_{\eta^{\sharp}}=\{0\}$. Hence applying the descending continuity property of Theorem 2.2 to inequality 4.1 , it follows

$$
C_{p}\left(\{0\}, B_{R^{\sharp}}\right) \leq C_{p}\left(S_{\varepsilon}, \Omega_{R}\right) .
$$

According to positivity rule for condenser $p$-capacity of points stated in Proposition 3.1 and to monotony properties stated in Theorem 2.2 inequality 4.2 provides no additional information:

(1) If $p \leq N$, the lower bound $C_{p}\left(\{0\}, B_{R^{\sharp}}\right)$ is null. 
(2) In the case $p>N$, one already knew that

$$
C_{p}\left(\{0\}, B_{R^{\sharp}}\right) \leq C_{p}\left(\{0\}, B_{R}\right) \leq C_{p}\left(S_{\varepsilon}, B\left(x_{0}, R\right)\right) \leq C_{p}\left(S_{\varepsilon}, \Omega_{R}\right) .
$$

Hence the anisotropy caused by the segment in the $p$-Laplace problem is not appropriately estimated by the symmetric rearrangement method applied hereabove.

4.3. From the point to the segment ? With notations of subsection 4.1, let us try to build an admissible solution $\bar{u}$ for the condenser $\left(S_{\varepsilon}, \Omega_{R}\right)$, when the length $\varepsilon$ is 'small enough'.

According to the descending continuity property, it holds

$$
\lim _{\varepsilon \rightarrow 0} C_{p}\left(S_{\varepsilon}, \Omega\right)=C_{p}\left(\left\{x_{0}\right\}, \Omega\right),
$$

Hence in the case $p>N$, an idea could be to define $\bar{u}$ starting from the radial function $s_{p, N}$ minimizing the energy of condenser $\left(\left\{x_{0}\right\}, B\left(x_{0}, R\right)\right)$. After Propositions 2.4 and 3.1 , for all $x \in \bar{B}\left(x_{0}, R\right)$, denoting $r=\left|x-x_{0}\right|$, it holds

$$
\left\{\begin{array}{l}
s_{p, N}(x)=s_{p, N}(r)=1-\left(\frac{r}{R}\right)^{\beta} \\
\left|\nabla s_{p, N}(x)\right|=\frac{\beta}{R^{\beta}} r^{\beta-1}, x \neq x_{0} .
\end{array}\right.
$$

Moreover

$$
\begin{aligned}
C_{p}\left(\left\{x_{0}\right\}, B\left(x_{0}, R\right)\right) & =\int_{B\left(x_{0}, R\right)}\left|\nabla s_{p, N}(x)\right|^{p} d x \\
& =A^{N-1} \frac{\beta^{p}}{R^{\beta p}} \int_{0}^{R} r^{(\beta-1) p+N-1} d r=A^{N-1} \beta^{p-1} R^{N-p},
\end{aligned}
$$

where exponent $(\beta-1) p+N-1=\beta-1=-\frac{N-1}{p-1} \in(-1,0)$.

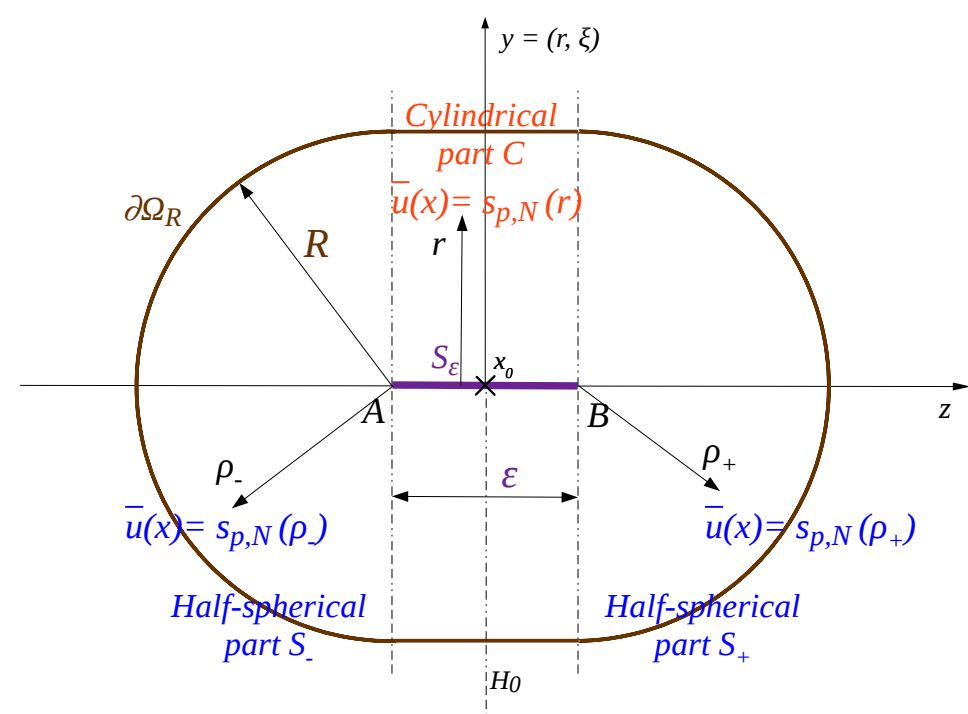

Figure 3. A poor candidate $\bar{u}$ for the condenser $\left(S_{\varepsilon}, \Omega_{R}\right)$.

Thus consider function $\bar{u}: \overline{\Omega_{R}} \rightarrow \mathbb{R}$ defined by:

$$
\begin{cases}\text { if } x \in \bar{S}_{-} \cap\{z<-\varepsilon / 2\} & \text { then } \bar{u}(x):=s_{p, N}\left(\rho_{-}\right) \text {with } \rho_{-}=|x-A|, \\ \text { if } x \in \bar{S}_{+} \cap\{z>\varepsilon / 2\} & \text { then } \bar{u}(x):=s_{p, N}\left(\rho_{+}\right) \text {with } \rho_{+}=|x-B|, \\ \text { if } x \in \bar{C} & \text { then } \bar{u}(x):=s_{p, N}(r) \text { with } r=|y| .\end{cases}
$$

as shown on figure 3 .

It is easy to check that $\bar{u}$ is continuous in $\overline{\Omega_{R}}$, and that $\bar{u}=0$ on $\partial \Omega_{R}$ and $\bar{u}=1$ on $S_{\varepsilon}$. As $\varepsilon>0$ is 'small enough', one could expect function $\bar{u}$ to be an admissible function for condenser $\left(S_{\varepsilon}, \Omega_{R}\right)$. 
Moreover one could expect the energy of $\bar{u}$ to provide an approximation of the capacity $C_{p}\left(S_{\varepsilon}, \Omega_{R}\right)$, as

$$
\int_{S_{-} \cup S_{+}}|\nabla \bar{u}(x)|^{p} d x=\int_{B\left(x_{0}, R\right)}\left|\nabla s_{p, N}(x)\right|^{p} d x=C_{p}\left(\left\{x_{0}\right\}, B\left(x_{0}, R\right)\right) .
$$

But the fact is function $\bar{u}$ is not even admissible for the condenser $\left(S_{\varepsilon}, \Omega_{R}\right)$. Calculating the energy of $\bar{u}$ in the cylindrical part $C$, it holds

$$
\int_{C}|\nabla \bar{u}(x)|^{p} d x=\int_{C}\left|\nabla s_{p, N}(x)\right|^{p} d x=\varepsilon A^{N-2} \frac{\beta^{p}}{R^{\beta p}} \int_{0}^{R} r^{(\beta-1) p} r^{N-2} d r=+\infty,
$$

as exponent $(\beta-1) p+N-2 \in(-2,-1)$.

Hence, despite the descending continuity property 4.3 in terms of energy, the solution minimizing the energy of the condenser, if it does exist, undergoes a sudden spatial reorganization when $\varepsilon$ shifts from 0 to a positive value. What matters primarily here is not the length of the perturbation $S_{\varepsilon}$, but the discontinuity of its dimension from 0 to 1 at the very moment $\varepsilon$ becomes positive. This example illustrates existing relationships between Hausdorff measures and capacities.

As a conclusion, when $p>N$, there is no hope to simply derive the asymptotic expansion of $C_{p}\left(S_{\varepsilon}, \Omega_{R}\right)$, even for $\varepsilon>0$ small enough, from the knowledge we have about $N$-dimensional spherical condensers.

\subsection{A lower-bound to the $p$-capacity of a segment.}

Proposition 4.2. With notations of subsection 4.1, the p-capacity of the equidistant condenser $\left(K_{\eta}, \Omega_{R}\right)$ admits the following lower-bound

$$
C_{p, N}\left(K_{\eta}, \Omega_{R}\right) \geq C_{p, N}(\eta, R)+\varepsilon C_{p, N-1}(\eta, R) .
$$

Proof. After subsection 2.2 and Proposition 2.3, we denote $u$ the admissible function minimizing the energy for the condenser $\left(K_{\eta}, \Omega_{R}\right)$. As

$$
C_{p, N}\left(K_{\eta}, \Omega_{R}\right)=\int_{C}|\nabla u|^{p} d x+\int_{S}|\nabla u|^{p} d x,
$$

we estimate separately each integral.

(1) In the cylindrical subset $C$, for all $a \in(-\varepsilon / 2, \varepsilon / 2)$, let $w_{a}$ be the restriction of $u$ to $H_{a} \cap\left(\overline{\Omega_{R} \backslash K_{\eta}}\right)$, that is

$$
w_{a}(y)=u(a, y), \quad \forall y \in \mathbb{R}^{N-1}, \eta \leq|y| \leq R .
$$

Due to the regularity of function $u, w_{a}$ is defined pointwise, continuous in $H_{a} \cap\left(\overline{\Omega_{R} \backslash K_{\eta}}\right)$ and $w_{a}$ admits a classical gradient in $H_{a} \cap\left(\Omega_{R} \backslash K_{\eta}\right)$.

Since $u$ is admissible for the condenser $\left(K_{\eta}, \Omega_{R}\right),|y|=\eta$ implies $w_{a}(y)=u(a, y)=1$ and $|y|=R$ implies $w_{a}(y)=u(a, y)=0$.

Moreover for all $y \in \mathbb{R}^{N-1}, \eta<|y|<R$ it holds:

$$
\left|\nabla_{y} w_{a}(y)\right|=\left|\nabla_{y} u(a, y)\right| \leq\left[\left|\nabla_{y} u(a, y)\right|^{2}+\left|\partial_{z} u(a, y)\right|^{2}\right]^{1 / 2}=|\nabla u(a, y)| .
$$

For a given $a \in(-\varepsilon / 2, \varepsilon / 2)$,

- if

$$
\int_{H_{a} \cap\left(\Omega_{R} \backslash K_{\eta}\right)}\left|\nabla_{y} w_{a}(y)\right|^{p} d y<+\infty,
$$

then $w_{a}$ is admissible to the $(N-1)$-dimensional condenser $\left(\bar{B}_{N-1}\left(x_{a}, \eta\right), B_{N-1}\left(x_{a}, R\right)\right)$. Thus:

$$
C_{p, N-1}(\eta, R) \leq \int_{H_{a} \cap\left(\Omega_{R} \backslash K_{\eta}\right)}\left|\nabla_{y} w_{a}(y)\right|^{p} d y \leq \int_{H_{a} \cap\left(\Omega_{R} \backslash K_{\eta}\right)}|\nabla u(a, y)|^{p} d y .
$$

- If

$$
\int_{H_{a} \cap\left(\Omega_{R} \backslash K_{\eta}\right)}\left|\nabla_{y} w_{a}(y)\right|^{p} d y=+\infty,
$$

inequality 4.5 obviously holds again. 
Integrating inequality 4.5 for all $a \in(-\varepsilon / 2, \varepsilon / 2)$, one obtains

$$
\varepsilon C_{p, N-1}(\eta, R) \leq \int_{C}|\nabla u(x)|^{p} d x .
$$

(2) Let $v$ be the function defined in $\bar{B}\left(x_{0}, R\right) \backslash B\left(x_{0}, \eta\right)$ which inherits the values taken by $u$ in the two half-spherical subsets $S_{ \pm}$. More precisely, for all $x \in \mathbb{R}^{N}, \eta \leq\left|x-x_{0}\right| \leq R$, we define

$$
\begin{cases}v(x):=u\left(B+x-x_{0}\right) & \text { if } z\left(x-x_{0}\right) \geq 0, \\ v(x):=u\left(A+x-x_{0}\right) & \text { if } z\left(x-x_{0}\right)<0 .\end{cases}
$$

Since $u$ is continuous in $\overline{\Omega_{R} \backslash K_{\eta}}$ and symmetric relatively to the hyperplane $H_{0}$, it follows that $v$ is continuous in $\bar{B}\left(x_{0}, R\right) \backslash B\left(x_{0}, \eta\right)$. Similarly $u \in L^{p}\left(\Omega_{R} \backslash K_{\eta}\right)$ implies that $v \in L^{p}\left(B\left(x_{0}, R\right) \backslash \bar{B}\left(x_{0}, \eta\right)\right)$.

For all $x \in\left(B\left(x_{0}, R\right) \backslash \bar{B}\left(x_{0}, \eta\right)\right) \cap\{z \neq 0\}$ it holds

$$
\begin{cases}\nabla v(x)=\nabla u\left(B+x-x_{0}\right) & \text { if } z\left(x-x_{0}\right)>0, \\ \nabla v(x)=\nabla u\left(A+x-x_{0}\right) & \text { if } z\left(x-x_{0}\right)<0 .\end{cases}
$$

Thus $\nabla u \in L^{p}\left(\Omega_{R} \backslash K_{\eta}\right)$ entails

$$
\nabla v \in L^{p}\left(\left(B\left(x_{0}, R\right) \backslash \bar{B}\left(x_{0}, \eta\right)\right) \cap\{z>0\}\right)
$$

and similarly

$$
\nabla v \in L^{p}\left(\left(B\left(x_{0}, R\right) \backslash \bar{B}\left(x_{0}, \eta\right)\right) \cap\{z<0\}\right) .
$$

Moreover, since $v$ is continuous in $\bar{B}\left(x_{0}, R\right) \backslash B\left(x_{0}, \eta\right)$ and thus has no jump accross $\{z=0\}$, the results about distribution derivatives (e.g. [36]) entail that the distribution $\nabla v$ defined in the domain $\left(B\left(x_{0}, R\right) \backslash \bar{B}\left(x_{0}, \eta\right)\right)$ can be identified to the vector field $\{\nabla v\}$ defined in

$$
\left(B\left(x_{0}, R\right) \backslash \bar{B}\left(x_{0}, \eta\right)\right) \cap\{z \neq 0\} .
$$

Hence $\nabla v \in L^{p}\left(B\left(x_{0}, R\right) \backslash \bar{B}\left(x_{0}, \eta\right)\right)$.

Lastly, as $u$ is admissible for the condenser $\left(K_{\eta}, \Omega_{R}\right)$, it holds $v(x)=1$ for all $x \in \mathbb{R}^{N}$, $\left|x-x_{0}\right|=\eta$ and $v(x)=0$ for all $x \in \mathbb{R}^{N},\left|x-x_{0}\right|=R$.

Therefore $v$ is an admissible function for the condenser $\left(\bar{B}\left(x_{0}, \eta\right), B\left(x_{0}, R\right)\right)$. It follows that

$$
C_{p, N}(\eta, R) \leq \int_{B\left(x_{0}, R\right) \backslash B\left(x_{0}, \eta\right)}|\nabla v(x)|^{p} d x=\int_{S}|\nabla u(x)|^{p} d x .
$$

Summing inequalities 4.6 and 4.7 yields the claimed result

$$
C_{p, N}\left(K_{\eta}, \Omega_{R}\right) \geq C_{p, N}(\eta, R)+\varepsilon C_{p, N-1}(\eta, R) .
$$

Thanks to equidistant condensers, we can now state the following lower-bound to the condenser $p$-capacity of a segment. Recall $C_{p, N}\left(\left\{x_{0}\right\}, B_{R}\right)$ (resp. $C_{p, N-1}\left(\left\{x_{0}\right\}, B_{R}\right)$ denotes the $p$-capacity of the point $\left\{x_{0}\right\}$ in the $N$-dimensional ball $B\left(x_{0}, R\right)$ (resp. the $p$-capacity of the point $\left\{x_{0}\right\}$ in the $(N-1)$-dimensional ball $\left.B_{N-1}\left(x_{0}, R\right)\right)$.

Theorem 4.3. Let $\Omega$ be a bounded domain of $\mathbb{R}^{N}$ and $x_{0} \in \Omega$. Let

$$
R:=\sup \left\{\left|y-x_{0}\right| ; y \in \Omega\right\} \in(0,+\infty) .
$$

Let $S_{\varepsilon}$ be a (closed) segment centered on the point $x_{0}$ and of length $\varepsilon>0$ such that $S_{\varepsilon} \subset \Omega$. Then the following lower-bound holds:

$$
C_{p, N}\left(S_{\varepsilon}, \Omega\right) \geq C_{p, N}\left(\left\{x_{0}\right\}, B_{R}\right)+\varepsilon C_{p, N-1}\left(\left\{x_{0}\right\}, B_{R}\right) .
$$

Proof. For all $\lambda>0$ and all $\eta$ such that $0<\eta<R$, inequality (4.4) of Proposition 4.2, applied to radiuses $\eta$ and $R+\lambda$, reads:

$$
C_{p, N}(\eta, R+\lambda)+\varepsilon C_{p, N-1}(\eta, R+\lambda) \leq C_{p, N}\left(K_{\eta}, \Omega_{R+\lambda}\right) .
$$

Three decreasing sequences of compacts are involved as follows:

$$
\bigcap_{\eta>0} \bar{B}\left(x_{0}, \eta\right)=\left\{x_{0}\right\}, \bigcap_{\eta>0} \bar{B}_{N-1}\left(x_{0}, \eta\right)=\left\{x_{0}\right\} \text { and } \bigcap_{\eta>0} K_{\eta}=S_{\varepsilon} .
$$


The descending continuity property of Theorem 2.2 hence implies that:

$$
\left\{\begin{array}{l}
\lim _{\eta \rightarrow 0} C_{p, N}\left(\bar{B}\left(x_{0}, \eta\right), B\left(x_{0}, R+\lambda\right)\right)=C_{p, N}\left(\left\{x_{0}\right\}, B\left(x_{0}, R+\lambda\right)\right) \\
\lim _{\eta \rightarrow 0} C_{p, N-1}\left(\bar{B}\left(x_{0}, \eta\right), B\left(x_{0}, R+\lambda\right)\right)=C_{p, N-1}\left(\left\{x_{0}\right\}, B\left(x_{0}, R+\lambda\right)\right) \\
\lim _{\eta \rightarrow 0} C_{p, N}\left(K_{\eta}, \Omega_{R+\lambda}\right)=C_{p, N}\left(S_{\varepsilon}, \Omega_{R+\lambda}\right) .
\end{array}\right.
$$

Therefore passing to the limit when $\eta \rightarrow 0$ in inequality 4.9 yields

$$
C_{p, N}\left(\left\{x_{0}\right\}, B_{R+\lambda}\right)+\varepsilon C_{p, N-1}\left(\left\{x_{0}\right\}, B_{R+\lambda}\right) \leq C_{p, N}\left(S_{\varepsilon}, \Omega_{R+\lambda}\right) .
$$

Moreover the inclusions $S_{\varepsilon} \subset \Omega \subset B\left(x_{0}, R+\lambda\right) \subset \Omega_{R+\lambda}$ hold. Hence the monotony property (ii) of Theorem 2.2 implies that

$$
C_{p, N}\left(S_{\varepsilon}, \Omega_{R+\lambda}\right) \leq C_{p, N}\left(S_{\varepsilon}, B\left(x_{0}, R+\lambda\right)\right) \leq C_{p, N}\left(S_{\varepsilon}, \Omega\right) .
$$

Gathering inequalities 4.10 and 4.11 entails

$$
C_{p, N}\left(\left\{x_{0}\right\}, B_{R+\lambda}\right)+\varepsilon C_{p, N-1}\left(\left\{x_{0}\right\}, B_{R+\lambda}\right) \leq C_{p, N}\left(S_{\varepsilon}, \Omega\right) .
$$

Lastly it follows from Theorem 3.1 that the mappings

$$
R>0 \mapsto C_{p, N}\left(\left\{x_{0}\right\}, B_{R}\right) \text { and } R \mapsto C_{p, N-1}\left(\left\{x_{0}\right\}, B_{R}\right)
$$

are continuous. Hence letting $\lambda$ tend towards 0 yields the claimed inequality.

Remark 4.4. The lower-bound of Theorem 4.3 is worth interpreting. Recall from Proposition 3.1 that the capacity of point $\left\{x_{0}\right\}$ in a bounded ball of $\mathbb{R}^{N}$ is positive if and only if $p>N$. Accordingly three cases are to be considered:

- If $N-1<p \leq N$, the point has a null $N$-dimensional condenser $p$-capacity but a positive $(N-1)$-dimensional condenser $p$-capacity. The inequality reads:

$$
\varepsilon C_{p, N-1}\left(\left\{x_{0}\right\}, B_{R}\right) \leq C_{p, N}\left(S_{\varepsilon}, \Omega\right)
$$

In particular, $C_{p, N}\left(S_{\varepsilon}, \Omega\right)>0$.

- If $p>N$, both capacities $C_{p, N}\left(\left\{x_{0}\right\}, B_{R}\right)$ and $C_{p, N-1}\left(\left\{x_{0}\right\}, B_{R}\right)$ are positive. Then again $C_{p, N}\left(S_{\varepsilon}, \Omega\right)>0$.

- If $p \leq N-1$, both capacities $C_{p, N}\left(\left\{x_{0}\right\}, B_{R}\right)$ and $C_{p, N-1}\left(\left\{x_{0}\right\}, B_{R}\right)$ are null.

Thus we can state a sufficient condition for the positivity of condenser $p$-capacities of segments.

Corollary 4.5. Let $S_{\varepsilon}$ be a segment of length $\varepsilon>0$ included in a bounded domain $\Omega \subset \mathbb{R}^{N}$. If $p>N-1$ then $C_{p, N}\left(S_{\varepsilon}, \Omega\right)>0$.

\subsection{Positivity of a condenser $p$-capacity of a segment in a bounded domain.}

Proposition 4.6. Let $S_{\varepsilon} \subset \Omega$ be a segment of length $\varepsilon>0$ centered on a point $x_{0}$. If $p \leq N-1$, then the condenser p-capacity of the segment $S_{\varepsilon}$ in the domain $\Omega$ is null, that is $C_{p, N}\left(S_{\varepsilon}, \Omega\right)=0$.

The proof is in section 5.2 on page 19 . According to Corollary 4.5 and to Proposition 4.6 , we can state the positivity rule for condenser $p$-capacities of segments.

Proposition 4.7. The condenser p-capacity of a segment $S_{\varepsilon}$ of length $\varepsilon>0$ included in a bounded domain $\Omega \subset \mathbb{R}^{N}$ is positive if and only if $p>N-1$.

Remark 4.8. It appeared according to Proposition 4.2 that the positivity of the condenser $p$-capacity of a segment in a $N$-dimensional bounded domain follows from the positivity of the condenser $p$-capacity of a point in a $(N-1)$-dimensional bounded domain.

Using equistant condensers derived from a plane rectangle, we may think of a similar proof to show that the positivity of the $p$-capacity of a plane rectangle in a $N$-dimensional bounded domain follows from the positivity of the $p$-capacity of a segment in a $(N-1)$-dimensional bounded domain, which happens when $p>(N-1)-1$. Such reasonings can be extended by induction to prove that the condenser $p$-capacity of a $k$-dimensional closed box in a $N$-dimensional bounded domain is positive as soon as $p>N-k$.

The cases of nullity for condenser capacity of a $k$-dimensional closed box seem to be more intricate to establish by means of equidistant condensers as the relationship between the capacity of a segment in a $N$-dimensional domain and the capacity of a point in a $(N-1)$-dimensional domain is not straightforward in the proof of Proposition 4.6 
4.6. Elliptical condensers. Let again a (closed) segment $S_{\varepsilon} \subset \mathbb{R}^{N}(N \geq 2)$, of length $\varepsilon>0$ and centered on a point $x_{0}$. Let $z$ be an axis passing through the point $x_{0}$ and parallel to the segment $S_{\varepsilon}$.

We first consider cylindrical coordinates $(z, y)=(z, r, \xi)$, with $z \in \mathbb{R}, y=r \xi \in \mathbb{R}^{N-1}, r \geq 0$ and $\xi \in S^{N-2}$. Then we move forward to the elliptic coordinates $(\mu, \nu, \xi)$ [39, 40] implicitely defined as follows for $\mu \in[0,+\infty), \nu \in[0, \pi]$ and $\xi \in S^{N-2}$,

$$
\begin{cases}z(\mu, \nu) & :=\frac{\varepsilon}{2} \cosh \mu \cos \nu, \\ r(\mu, \nu) & :=\frac{\varepsilon}{2} \sinh \mu \sin \nu, \\ \xi & :=\xi\end{cases}
$$

In particular $S_{\varepsilon}=\{\mu=0, \nu \in[0, \pi]\}$. Hence the segment $S_{\varepsilon}$ is considered here as the limit of an ellipsoid which eccentricity tends toward 1 when $\mu \rightarrow 0$.

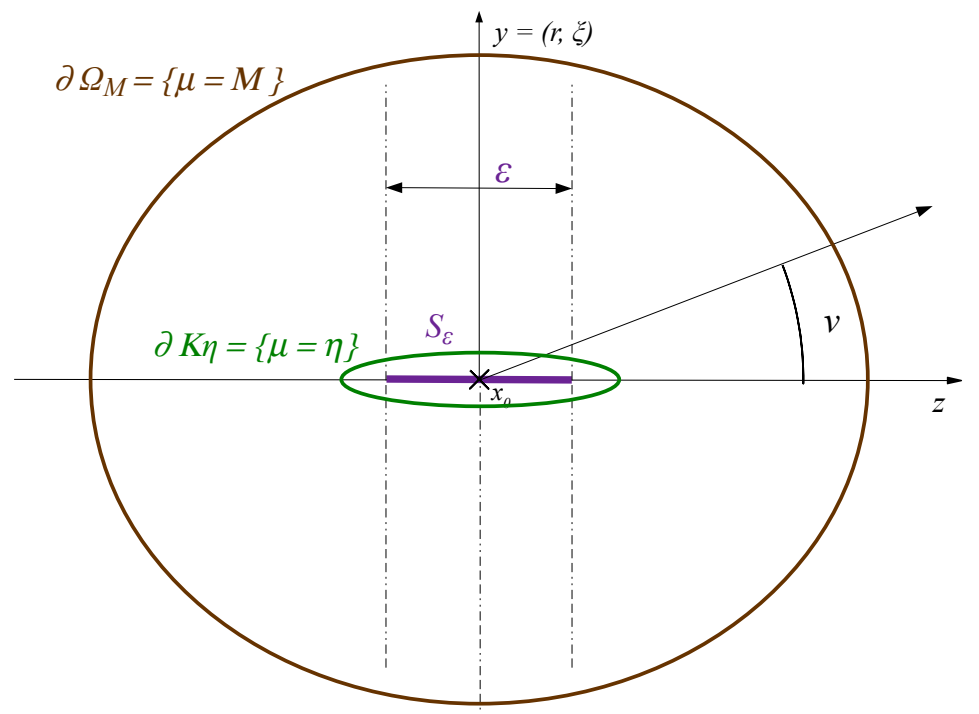

FiguRE 4. An elliptical condenser $\left(K_{\eta}, \Omega_{M}\right)$.

Looking at figure 4 we set

Definition 4.9. Let $0<\eta<M$. Let the bounded domain

$$
\Omega_{M}:=\left\{x=(\mu, \nu, \xi) \in \mathbb{R}^{N} ; 0 \leq \mu<M, \nu \in[0, \pi], \xi \in S^{N-2}\right\}
$$

and the compact set

$$
K_{\eta}:=\left\{x=(\mu, \nu, \xi) \in \mathbb{R}^{N} ; 0 \leq \mu \leq \eta, \nu \in[0, \pi], \xi \in S^{N-2}\right\} .
$$

We say that $\left(K_{\eta}, \Omega_{M}\right)$ is an elliptical condenser derived from the segment $S_{\varepsilon}$.

Obviously the inclusions $S_{\varepsilon} \subset K_{\eta} \subset \Omega_{M}$ hold for all $0<\eta<M$. Moreover it holds $\bigcap_{\eta>0} K_{\eta}=S_{\varepsilon}$. In comparison with equidistant condensers though, letting $\eta \rightarrow 0$ will not be sufficient to approximate asymptotically, when $\varepsilon \rightarrow 0$, the condenser made of the segment $S_{\varepsilon}$ within a given bounded domain $\Omega$. Due to 4.12 indeed, for a given $M>0, \Omega_{M} \rightarrow\left\{x_{0}\right\}$ when $\varepsilon \rightarrow 0$. So that we shall have to choose some appropriate $M(\varepsilon) \rightarrow+\infty$ to approximate a given domain $\Omega$ by $\Omega_{M(\varepsilon)}$ when $\varepsilon \rightarrow 0$.

Lemma 4.10. Let $R>\varepsilon / 2>0$ and let

$$
\left\{\begin{array}{l}
M^{\prime}:=\log \left(2 R / \varepsilon+\sqrt{1+4 R^{2} / \varepsilon^{2}}\right) \\
M^{\prime \prime}:=\log \left(2 R / \varepsilon+\sqrt{-1+4 R^{2} / \varepsilon^{2}}\right) .
\end{array}\right.
$$

Let $K$ a compact of $\mathbb{R}^{N}$ such that $K \subset \Omega_{M^{\prime \prime}}$. Then it holds

$$
C_{p}\left(K, \Omega_{M^{\prime}}\right) \leq C_{p}\left(K, B_{R}\right) \leq C_{p}\left(K, \Omega_{M^{\prime \prime}}\right) .
$$


In particular, for all $\eta$ such that $0<\eta<M^{\prime \prime}$, it holds

$$
C_{p}\left(K_{\eta}, \Omega_{M^{\prime}}\right) \leq C_{p}\left(K_{\eta}, B_{R}\right) \leq C_{p}\left(K_{\eta}, \Omega_{M^{\prime \prime}}\right) .
$$

and

$$
C_{p}\left(S_{\varepsilon}, \Omega_{M^{\prime}}\right) \leq C_{p}\left(S_{\varepsilon}, B_{R}\right) \leq C_{p}\left(S_{\varepsilon}, \Omega_{M^{\prime \prime}}\right)
$$

Proof. It follows from 4.12 that

$$
B_{\frac{\varepsilon}{2} \sinh M} \subset \Omega_{M} \subset B_{\frac{\varepsilon}{2}} \cosh M, \quad \forall M>0 .
$$

Moreover the definition (4.13) of $M^{\prime}$ and $M^{\prime \prime}$ can be solved into

$$
R=\frac{\varepsilon}{2} \sinh M^{\prime}=\frac{\varepsilon}{2} \cosh M^{\prime \prime} .
$$

Hence plugging $M=M^{\prime}$ (resp. $M=M^{\prime \prime}$ ) into (4.17), one obtains

$$
\Omega_{M^{\prime \prime}} \subset B_{R} \subset \Omega_{M^{\prime}} \text {. }
$$

Thus the monotony property (ii) of Theorem 2.2 implies

$$
C_{p}\left(K, \Omega_{M^{\prime}}\right) \leq C_{p}\left(K, B_{R}\right) \leq C_{p}\left(K, \Omega_{M^{\prime \prime}}\right)
$$

which the claimed inequality (4.14).

In particular, chosing $K=K_{\eta}$ (resp. $K=S_{\varepsilon}$ ), it follows 4.15) (resp.4.16)).

4.7. The condenser 2-capacity of a segment. In the harmonic case $p=2$, according to Theorem 4.7. the condenser capacity of a segment is positive in a bounded domain of $\mathbb{R}^{2}$ while it is null in higher dimensions $N \geq 3$.

Proposition 4.11. Let $0<\varepsilon / 2<R$. Let $S_{\varepsilon}$ a (closed) segment centered on a point $x_{0}$ and of length $\varepsilon$ and let $B_{R}=B\left(x_{0}, R\right)$ be both subsets of $\mathbb{R}^{2}$. Then the following inequalities hold:

$$
\frac{2 \pi}{\log \left(2 R / \varepsilon+\sqrt{1+4 R^{2} / \varepsilon^{2}}\right)} \leq C_{2}\left(S_{\varepsilon}, B_{R}\right) \leq \frac{2 \pi}{\log \left(2 R / \varepsilon+\sqrt{-1+4 R^{2} / \varepsilon^{2}}\right)} .
$$

Proof. We calculate $C_{p}\left(K_{\eta}, \Omega_{M}\right)$ applying Proposition 2.3. Due to the symmetry of revolution relatively to the $z$-axis, the searched solution does not depend upon coordinate $\xi \in S^{N-2}$. Thus in elliptic coordinates, the Laplacian operator applied to $u$ reads:

$$
\Delta u(\mu, \nu)=\frac{4}{\varepsilon^{2}} \frac{\partial_{\mu \mu} u+\partial_{\nu \nu} u}{\sinh ^{2} \mu+\sin ^{2} \nu} .
$$

Hence Problem (2.3) becomes

$$
\begin{cases}\partial_{\mu \mu} u+\partial_{\nu \nu} u=0 & \text { in } \Omega_{M} \backslash K_{\eta} \\ u(\eta, \nu)=1 & \forall \nu \in[0, \pi] \\ u(M, \nu)=0 & \forall \nu \in[0, \pi]\end{cases}
$$

The separation of variables yields

$$
u(\mu, \nu)=\frac{M-\mu}{M-\eta}, \quad \forall \mu \in[\eta, M], \forall \nu \in[0, \pi] .
$$

Then a simple calculation provides

$$
|\nabla u|^{2}=\frac{4}{\varepsilon^{2}\left(\sinh ^{2} \mu+\sin ^{2} \nu\right)} \frac{1}{(M-\eta)^{2}} .
$$

Since

$$
|\operatorname{det} D(z, r, \xi) / D(\mu, \nu, \xi)|=(\varepsilon / 2)^{2}\left(\sinh ^{2} \mu+\sin ^{2} \nu\right),
$$

the change of variables yields

$$
C_{2}\left(K_{\eta}, \Omega_{M}\right)=\int_{\Omega_{M} \backslash K_{\eta}}|\nabla u|^{2}=\frac{2 \pi}{M-\eta} .
$$

When $\eta \rightarrow 0$, according to the descending continuity stated in Theorem 2.2 it follows that

$$
C_{2}\left(S_{\varepsilon}, \Omega_{M}\right)=\frac{2 \pi}{M} .
$$


Applying equality 4.22 for $M=M^{\prime}$ (resp. $M=M^{\prime \prime}$ ) and inequality 4.16) of Lemma 4.10 one obtains

$$
\frac{2 \pi}{\log \left(2 R / \varepsilon+\sqrt{1+4 R^{2} / \varepsilon^{2}}\right)} \leq C_{2}\left(S_{\varepsilon}, B_{R}\right) \leq \frac{2 \pi}{\log \left(2 R / \varepsilon+\sqrt{-1+4 R^{2} / \varepsilon^{2}}\right)}
$$

which are the claimed inequalities 4.20 .

Corollary 4.12. Let $\Omega$ a bounded domain $\subset \mathbb{R}^{2}$ and $x_{0}$ a point of $\Omega$. Let $S_{\varepsilon} \subset \mathbb{R}^{2}$ be a segment centered on a point $x_{0}$ and of length $\varepsilon>0$. Then for $\varepsilon$ small enough it holds

$$
C_{2}\left(S_{\varepsilon}, \Omega\right)=\frac{-2 \pi}{\log \varepsilon}+o\left(\frac{-1}{\log \varepsilon}\right) .
$$

Proof. Let $R_{2}>R_{1}>0$ such that $B\left(x_{0}, R_{1}\right) \subset \Omega \subset B\left(x_{0}, R_{2}\right)$. Let $0<\varepsilon<R_{1}$. Thus $S_{\varepsilon} \subset B\left(x_{0}, R_{1}\right)$. According to monotony property (ii) of Theorem 2.2 it holds

$$
C_{2}\left(S_{\varepsilon}, B\left(x_{0}, R_{2}\right)\right) \leq C_{2}\left(S_{\varepsilon}, \Omega\right) \leq C_{2}\left(S_{\varepsilon}, B\left(x_{0}, R_{1}\right)\right) .
$$

Applying inequalities 4.20 of Proposition 4.11 for $R=R_{1}$ (resp. $R=R_{2}$ ), one obtains

$$
C_{2}\left(S_{\varepsilon}, B\left(x_{0}, R_{1}\right)\right) \leq \frac{2 \pi}{\log \left(2 R_{1} / \varepsilon+\sqrt{-1+4 R_{1}^{2} / \varepsilon^{2}}\right)}
$$

and

$$
\frac{2 \pi}{\log \left(2 R_{2} / \varepsilon+\sqrt{1+4 R_{2}^{2} / \varepsilon^{2}}\right)} \leq C_{2}\left(S_{\varepsilon}, B\left(x_{0}, R_{2}\right)\right) .
$$

Hence

$$
\frac{2 \pi}{\log \left(2 R_{2} / \varepsilon+\sqrt{1+4 R_{2}^{2} / \varepsilon^{2}}\right)} \leq C_{2}\left(S_{\varepsilon}, \Omega\right) \leq \frac{2 \pi}{\log \left(2 R_{1} / \varepsilon+\sqrt{-1+4 R_{1}^{2} / \varepsilon^{2}}\right)} .
$$

The claimed asymptotic expansion (4.24) follows.

Remark 4.13. - Asymptotic expansion (4.24) is a topological asymptotic expansion in the sense of (1.1), here obtained with Dirichlet boundary condition. For the study of the perturbation of the Laplace equation in 2D by a Neumann homogeneous boundary condition on a segment, see [1].

- According to [15, it is already known that, in the case of the Laplace equation with Dirichlet boundary condition in 2D, the first order of the topological expansion does not depend on the shape of the obstacle, in the case of obstacles with non-empty interiors.

After Proposition 2.4 in the case $p=N=2$ and for spherical condensers, it holds

$$
C_{2}\left(B_{\varepsilon / 2}, B_{R}\right)=\frac{2 \pi}{\log (2 R / \varepsilon)}=\frac{-2 \pi}{\log \varepsilon}+o\left(\frac{-1}{\log \varepsilon}\right) .
$$

It is noticeable that the first term $-2 \pi / \log \varepsilon$ of the asymptotic expansion (4.24) of $C_{2}\left(S_{\varepsilon}, B_{R}\right)$ is the same as the first term of the expansion of $C_{2}\left(B_{\varepsilon / 2}, B_{R}\right)$. Thus according to monotony property $(i)$ of Theorem 2.2 , it holds

$$
C_{2}\left(K_{\varepsilon}, B_{R}\right)=\frac{-2 \pi}{\log \varepsilon}+o\left(\frac{-1}{\log \varepsilon}\right) \text {, for all compact } K_{\varepsilon}, S_{\varepsilon} \subset K_{\varepsilon} \subset B_{\varepsilon / 2} .
$$

Hence the topological gradient does not depend on the shape of the obstacle $K_{\varepsilon}$, even when $K_{\varepsilon}$ is the segment $S_{\varepsilon}$. It only depends on its size. Therefore the topological gradient of the 2 -capacity in $2 \mathrm{D}$ is not a appropriate tool for sorting out curves and obstacles with non empty interior.

One could try to overcome this drawback, either by considering different values of $p$ or by trying to sort out shapes according to the second order of the asymptotic expansion, that is considering a topological hessian.

Though we do not know yet the asymptotic expansion of $C_{p}\left(S_{\varepsilon}, B_{R}\right)$ in higher dimensions $N \geq 3$, the same difficulty might also arise for $p=N \geq 3$ according to Remark 2.6 on page 7 . 
Remark 4.14. In the harmonic case $p=2$, equation 2.3 can be explicitely solved as its enjoys separable variables in elliptic coordinates. However there are some clues that, even when $p \neq 2$, elliptical condensers may be applied for further estimations of the $p$-capacity of a segment $S_{\varepsilon}$ in a given domain $\Omega$. As usual, $S_{\varepsilon}$ has first to be approximated by an ellipsoid $K_{\eta}$, with $\eta$ 'small enough'.

Elliptic coordinates seem appropriate for segments in the sense that angular coordinate $\nu$ so to speak makes the dimension in which operates the $p$-Laplace equation, continuously change from $N$ for $\nu=0$ to $(N-1)$ for $\nu=\pi / 2$ and then back to $N$ for $\nu=\pi$.

Furthermore the geometry of the problem is simplified with elliptical condensers as solutions and integrals are to be calculated on the rectangle $\mathcal{R}:=[\eta, M] \times[0, \pi]$.

After a change of variables, one can rewrite minimization problem 2.5 in elliptic coordinates $(\mu, \nu) \in \mathcal{R}$. Let the weight $E$ given by

$$
E(\mu, \nu):=\frac{(\sinh \mu \sin \nu)^{N-2}}{\left(\sinh ^{2} \mu+\sin ^{2} \nu\right)^{\frac{p-2}{2}}}, \forall \mu>0, \forall \nu \in[0, \pi],
$$

and the functional

$$
J(v):=A^{N-2}\left(\frac{\varepsilon}{2}\right)^{N-p} \int_{\mathcal{R}} E(\mu, \nu)|\nabla v(\mu, \nu)|^{p} \mathrm{~d} \mu \mathrm{d} \nu .
$$

Then the capacity $C_{p}\left(K_{\eta}, \Omega_{M}\right)$ may be obtained by minimizing functional $J$ in an appropriate set of functions $v: \mathcal{R} \rightarrow \mathbb{R}$, such that $E^{\frac{1}{p}} \nabla v \in L^{p}(\mathcal{R})$ and such that $v(\eta, \nu)=1$ and $v(M, \nu)=0$, for all $\nu \in[0, \pi]$.

\section{Proofs}

5.1. Proof of Proposition 2.4. We prove Proposition 2.4 by solving problem 2.3 for the spherical condenser and then applying Proposition 2.3 Hence consider the following problem:

$$
\begin{cases}-\Delta_{p} s_{p, N}=0 & \text { in } B_{R} \backslash \bar{B}_{\varepsilon} \\ s_{p, N}=1 & \text { on } \partial B_{\varepsilon} \\ s_{p, N}=0 & \text { on } \partial B_{R}\end{cases}
$$

As recalled in subsection 2.2 on page 3 there exists a unique solution $s_{p, N} \in W^{1, p}\left(B_{R} \backslash \bar{B}_{\varepsilon}\right)$ to problem 5.1. We look for a solution $s_{p, N}$ with radial symmetry, that is:

$$
s_{p, N}(x)=f(r), \quad \forall x \in B_{R} \backslash \bar{B}_{\varepsilon}, r=\left|x-x_{0}\right| .
$$

Assuming that function $f$ is regular enough, a calculation in spherical coordinates yields:

$$
\Delta_{p} s_{p, N}= \pm\left(f^{\prime}(r)\right)^{(p-1)}\left[(p-1) f^{\prime \prime}(r)+\frac{N-1}{r} f^{\prime}(r)\right] .
$$

We assume that there is no point in $\bar{B}_{R} \backslash B_{\varepsilon}$ such that $f^{\prime}(r)=0$.

(1) In the case $p=N$, it follows that $f^{\prime}(r)=C / r$. Then applying the Dirichlet conditions of problem (5.1) yields:

$$
s_{p, N}(x)=f(r)=\frac{\log (R / r)}{\log (R / \varepsilon)}, \quad \forall x \in B_{R} \backslash \bar{B}_{\varepsilon}
$$

and

$$
\left|\nabla s_{p, N}(x)\right|=\left|f^{\prime}(r)\right|=\frac{1}{\log (R / \varepsilon)} \frac{1}{r}, \quad \forall x \in B_{R} \backslash \bar{B}_{\varepsilon} .
$$

Conversely it is easy to check that there is no point in $\bar{B}_{R} \backslash B_{\varepsilon}$ such that $\left|\nabla s_{p, N}(x)\right|=0$ as defined in (5.4) and that the function $s_{p, N}$ stated by (5.3) is the unique solution to problem (5.1).

Applying Proposition 2.3 the capacity of the spherical condenser $\left(\bar{B}_{\varepsilon}, B_{R}\right)$ reads:

$$
C_{p}(\varepsilon, R)=\frac{A^{N-1}}{(\log (R / \varepsilon))^{p}} \int_{\varepsilon}^{R} \frac{r^{N-1}}{r^{p}} d r=A^{N-1}[\log (R / \varepsilon)]^{1-p} .
$$

The claimed asymptotic expansion follows when $\varepsilon \rightarrow 0$. 
(2) In the case $p \neq N$, it follows from 5.2 that $f^{\prime}(r)=C r^{\beta-1}$. Applying the Dirichlet conditions of problem $(5.1)$ yields

$$
s_{p, N}(x)=f(r)=\frac{R^{\beta}-r^{\beta}}{R^{\beta}-\varepsilon^{\beta}}, \quad \forall x \in B_{R} \backslash \bar{B}_{\varepsilon},
$$

and

$$
\left|\nabla s_{p, N}(x)\right|=\left|f^{\prime}(r)\right|=\left|\frac{\beta}{R^{\beta}-\varepsilon^{\beta}}\right| \quad r^{\beta-1}, \quad \forall x \in B_{R} \backslash \bar{B}_{\varepsilon} .
$$

Conversely it is easy to check that there is no point in $\bar{B}_{R} \backslash B_{\varepsilon}$ such that $\left|\nabla s_{p, N}(x)\right|=0$ as defined in equation (5.6) and that the function $s_{p, N}$ defined by equation (5.5) is the unique solution to problem (5.1).

Applying Proposition 2.3 the capacity of the spherical condenser $\left(\bar{B}_{\varepsilon}, B_{R}\right)$ reads:

$$
C_{p}\left(\bar{B}_{\varepsilon}, B_{R}\right)=A^{N-1}\left|\frac{\beta}{R^{\beta}-\varepsilon^{\beta}}\right|^{p} \int_{\varepsilon}^{R} r^{N-1+p(\beta-1)} d r=A^{N-1}|\beta|^{p-1}\left|R^{\beta}-\varepsilon^{\beta}\right|^{1-p} .
$$

The claimed asymptotic expansions follow, when $\varepsilon \rightarrow 0$, dealing separately with the two cases $p>N$ and $p<N$.

Note that (5.5), (5.6) and (5.7) also hold in the case $N=1<p$, with the convention $A^{0}=2$.

5.2. Proof of Proposition 4.6. Let $\Omega^{c}:=\mathbb{R}^{N} \backslash \Omega$. Since $\Omega$ is bounded there exists $M>0$ such that $\Omega \subset B\left(x_{0}, M / 2\right)$. Then $S_{\varepsilon}$ and $\Omega^{c} \cap \bar{B}\left(x_{0}, M\right)$ are compact sets such that

$$
S_{\varepsilon} \cap\left(\Omega^{c} \cap \bar{B}\left(x_{0}, M\right)\right)=\emptyset .
$$

Therefore due to the continuity of the distance, there exist $x^{\prime} \in S_{\varepsilon}$ and $x^{\prime \prime} \in \Omega^{c} \cap \bar{B}\left(x_{0}, M\right)$ such that:

$$
\left|x^{\prime}-x^{\prime \prime}\right|=\min \left\{\left|x_{1}-x_{2}\right| ; x_{1} \in S_{\varepsilon} \text { and } x_{2} \in \Omega^{c} \cap \bar{B}\left(x_{0}, M\right)\right\}>0 .
$$

Let $R:=\left|x^{\prime}-x^{\prime \prime}\right| / 2$ and $\Omega_{R}=\left\{x \in \mathbb{R}^{N} ; d\left(S_{\varepsilon}, x\right)<R\right\}$. It holds $S_{\varepsilon} \subset \Omega_{R} \subset \Omega$. Hence according to monotony property (ii) stated in Theorem 2.2 , it follows that

$$
C_{p, N}\left(S_{\varepsilon}, \Omega\right) \leq C_{p, N}\left(S_{\varepsilon}, \Omega_{R}\right) .
$$

Therefore it suffices to prove that $C_{p, N}\left(S_{\varepsilon}, \Omega_{R}\right)=0$. According to the descending continuity property of Theorem 2.2 it holds

$$
C_{p, N}\left(S_{\varepsilon}, \Omega_{R}\right)=\lim _{\eta \rightarrow 0} C_{p}\left(K_{\eta}, \Omega_{R}\right) .
$$

Hence it suffices to prove that

$$
\lim _{\eta \rightarrow 0} C_{p}\left(K_{\eta}, \Omega_{R}\right)=0
$$

(1) We first prove $(5.8)$ in the case $p<N-1$.

Recall $s_{p, N}$ denotes the admissible function minimizing energy for the $N$-dimensional spherical condenser $\left(B_{\eta}, B_{R}\right)$, that is

$$
C_{p, N}\left(B_{\eta}, B_{R}\right)=\int_{B_{R} \backslash B_{\eta}}\left|\nabla s_{p, N}\right|^{p} d x .
$$

Let the function $v: \overline{\Omega_{R} \backslash K_{\eta}} \rightarrow \mathbb{R}$ defined by:

$$
\begin{cases}\text { if } x \in \bar{S}_{-} \cap\{z<-\varepsilon / 2\} & \text { then } v(x):=s_{p, N}\left(\rho_{-}\right) \text {with } \rho_{-}=|x-A|, \\ \text { if } x \in \bar{S}_{+} \cap\{z>\varepsilon / 2\} & \text { then } v(x):=s_{p, N}\left(\rho_{+}\right) \text {with } \rho_{+}=|x-B|, \\ \text { if } x \in \bar{C} & \text { then } v(x):=s_{p, N}(r) \text { with } r=|y| .\end{cases}
$$

as shown on figure 5

It is easy to check that $v$ is continuous in $\overline{\Omega_{R} \backslash K_{\eta}}$, that $v \in W^{1, p}\left(\Omega_{R} \backslash K_{\eta}\right)$ and that $v=0$ on $\partial \Omega_{R}$ and $v=1$ on $\partial K_{\eta}$. Thus $v$ is admissible for the condenser $\left(K_{\eta}, \Omega_{R}\right)$. Hence

$$
C_{p, N}\left(K_{\eta}, \Omega_{R}\right) \leq \int_{C \cup S}|\nabla v|^{p} d x .
$$

Thus to prove 5.8 it suffices to prove that

$$
\lim _{\eta \rightarrow 0} \int_{C \cup S}|\nabla v|^{p} d x=0 .
$$




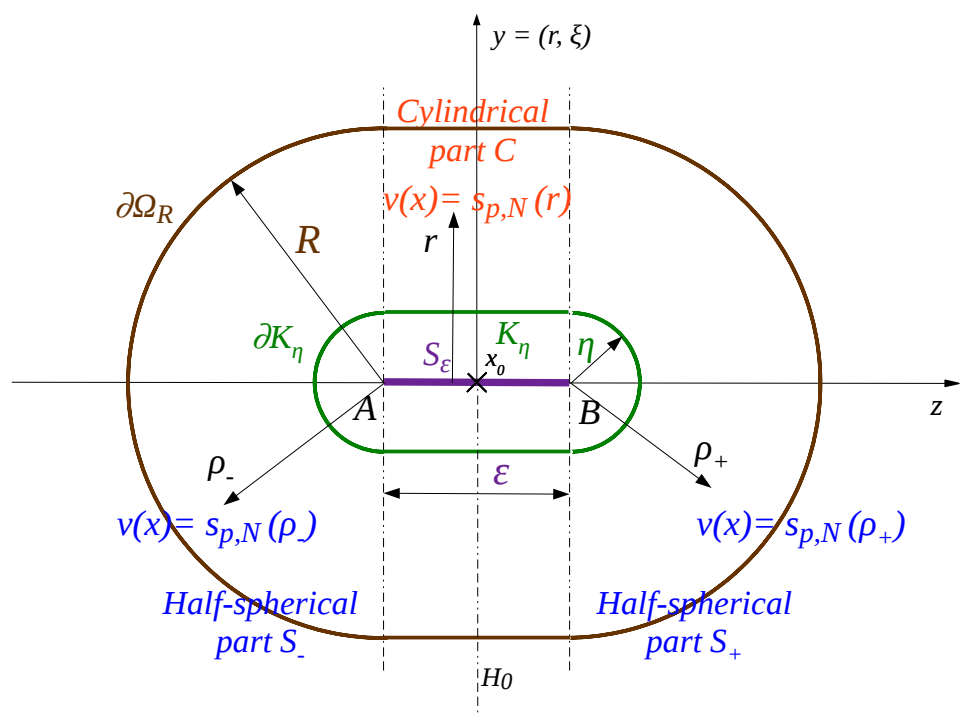

FIGURE 5. The admissible function $v$ for the condenser $\left(K_{\eta}, \Omega_{R}\right)$.

By definition of $v$ it holds

$$
\int_{S}|\nabla v|^{p} d x=C_{p, N}\left(B_{\eta}, B_{R}\right) .
$$

Since $p<N$, it follows from the descending continuity of Theorem 2.2 and from Proposition 3.1 that

$$
\lim _{\eta \rightarrow 0} \int_{S}|\nabla v|^{p} d x=\lim _{\eta \rightarrow 0} C_{p, N}\left(B_{\eta}, B_{R}\right)=C_{p, N}\left(\left\{x_{0}\right\}, B_{R}\right)=0 .
$$

Furthermore an integration in cylindrical coordinates in $C$ yields:

$$
\int_{C}|\nabla v|^{p} d x=\varepsilon A^{N-2} \int_{\eta}^{R}\left|\partial_{r} s_{p, N}(r)\right|^{p} r^{N-2} d r
$$

As $p<N-1$ after Proposition 2.4 it holds

$$
\left|\partial_{r} s_{p, N}(r)\right|=\left[-\beta /\left(\eta^{\beta}-R^{\beta}\right)\right] r^{\beta-1} .
$$

Hence

$$
\int_{\eta}^{R}\left|\partial_{r} s_{p, N}(r)\right|^{p} r^{N-2} d r=\left[\frac{-\beta}{\eta^{\beta}-R^{\beta}}\right]^{p} \frac{\eta^{\beta-1}-R^{\beta-1}}{1-\beta} .
$$

Since $\beta<0$, when $\eta$ tends towards 0 , the integral is equivalent to

$$
\frac{(-\beta)^{p}}{1-\beta} \eta^{\beta-1-p \beta}
$$

with $\beta-1-p \beta=N-p-1>0$. It follows that

$$
\lim _{\eta \rightarrow 0} \int_{\eta}^{R}\left|\partial_{r} s_{p, N}(r)\right|^{p} r^{N-2} d r=0
$$

and that $\lim _{\eta \rightarrow 0} \int_{C}|\nabla v|^{p} d x=0$.

This completes the proof of $(5.9)$ and thus the proof of $(5.8)$ in the case $p<N-1$.

(2) We now prove 5.8$)$ in the case $p=N-1$.

Recall $s_{p, N-1}$ denotes the admissible function minimizing energy for the $(N-1)$-dimensional spherical condenser $\left(B_{N-1}(\eta), B_{N-1}(R)\right)$, that is

$$
C_{p, N-1}\left(B_{N-1}(\eta), B_{N-1}(R)\right)=\int_{B_{N-1}(R) \backslash B_{N-1}(\eta)}\left|\nabla s_{p, N-1}\right|^{p} d y,
$$




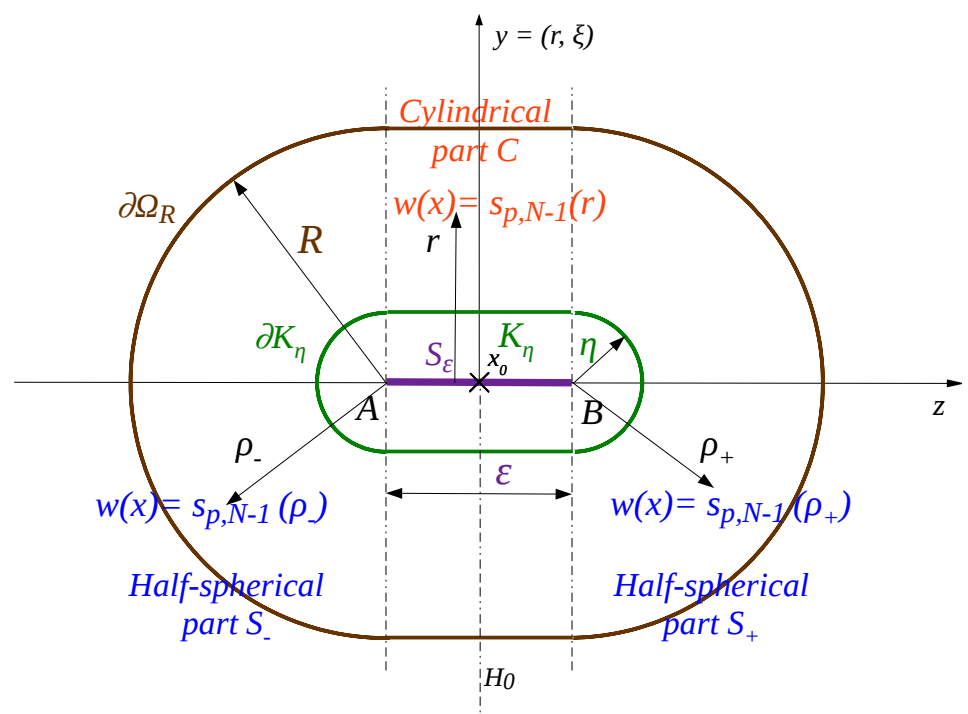

FiguRE 6. The admissible function $w$ for the condenser $\left(K_{\eta}, \Omega_{R}\right)$.

where $d y$ denotes the Lebesgue measure in $\mathbb{R}^{N-1}$.

Let the function $w: \Omega_{R} \backslash K_{\eta} \rightarrow \mathbb{R}$ defined by:

$$
\begin{cases}\text { if } x \in \bar{C} & \text { then } w(x):=s_{p, N-1}(r) \text { with } r=|y|, \\ \text { if } x \in \bar{S}_{-} \cap\{z<-\varepsilon / 2\} & \text { then } w(x):=s_{p, N-1}\left(\rho_{-}\right) \text {with } \rho_{-}=|x-A|, \\ \text { if } x \in \bar{S}_{+} \cap\{z>\varepsilon / 2\} & \text { then } w(x):=s_{p, N-1}\left(\rho_{+}\right) \text {with } \rho_{+}=|x-B| .\end{cases}
$$

as shown on figure 6

As for function $v$, it is easy to check that $w$ is an admissible function for the $N$-dimensional condenser $\left(K_{\eta}, \Omega_{R}\right)$. Hence

$$
C_{p, N}\left(K_{\eta}, \Omega_{R}\right) \leq \int_{C \cup S}|\nabla w|^{p} d x .
$$

Thus to prove (5.8), it suffices to prove that

$$
\lim _{\eta \rightarrow 0} \int_{C \cup S}|\nabla w|^{p} d x=0 .
$$

By definition of $w$ it holds

$$
\int_{C}|\nabla w|^{p} d x=\varepsilon C_{p, N-1}\left(B_{N-1}(\eta), B_{N-1}(R)\right) .
$$

Since $p=N-1$, it follows from the descending continuity of Theorem 2.2 and from Theorem 3.1 that

$$
\begin{aligned}
\lim _{\eta \rightarrow 0} \int_{C}|\nabla w|^{p} d x & =\varepsilon \lim _{\eta \rightarrow 0} C_{p, N-1}\left(B_{N-1}(\eta), B_{N-1}(R)\right) \\
& =\varepsilon C_{p, N-1}\left(\left\{x_{0}\right\}, B_{N-1}(R)\right)=0 .
\end{aligned}
$$

Furthermore an integration in spherical coordinates in $S$ yields:

$$
\int_{S}|\nabla w|^{p} d x=A^{N-1} \int_{\eta}^{R}\left|\partial_{\rho} s_{p, N-1}(\rho)\right|^{p} \rho^{N-1} d \rho .
$$

As $p=N-1$, the gradient reads

$$
\left|\partial_{\rho} s_{p, N-1}(\rho)\right|=\frac{1}{\log (R / \eta)} \frac{1}{\rho} .
$$


Hence

Therefore

$$
\int_{\eta}^{R}\left|\partial_{\rho} s_{p, N-1}(\rho)\right|^{p} \rho^{N-1} d \rho=|\log (R / \eta)|^{-p}(R-\eta) .
$$

$$
\lim _{\eta \rightarrow 0} \int_{\eta}^{R}\left|\partial_{\rho} s_{p, N-1}(\rho)\right|^{p} \rho^{N-1} d \rho=0
$$

and thus

$$
\lim _{\eta \rightarrow 0} \int_{S}|\nabla w|^{p} d x=0 .
$$

This completes the proof of (5.10) and thus the proof (5.8) in the case $p=N-1$.

\section{CONCLUSiOns AND PROSPECTS}

In this article, we consider compact sets with positive condenser $p$-capacities and we provide estimates and asymptotic expansions of such capacities. The emphasis is put on capacities of points and of segments.

Our main contributions deal with condenser $p$-capacities of segments. Nevertheless it is fruitful to first study in detail condenser capacities which obstacle either has a non empty interior or is a point.

As premilinary results, we show that one can calculate a condenser $p$-capacity by solving a $p$-Laplace equation with Dirichlet boundary condition, when the boundaries of the condenser are smooth. We provide asymptotic bounds to condenser $p$-capacities when the obstacle has a non-empty interior. As for condenser capacities of points, we provide estimates, asymptotic approximations and positivity cases. We study the speed of the descending continuity property, i.e. the ability to approximate the capacity of a point by that of a ball with a 'small enough' radius. We show that the speed of convergence is slow.

We then move on to condenser $p$-capacities of segments. Introducing equidistant condensers, we illustrate in two ways the strong anisotropy caused by a segment acting as an obstacle in the $p$-Laplace equation. First we show that the Pólya-Szegö rearrangement inequality for Dirichlet type integrals fails to provide a valuable lower-bound to the capacity of a segment. Secondly, in the case $p>N$, we show that, however small the length of a segment may be, one cannot derive an admissible function for the segment by simply extending the function minimizing the energy for a point.

Our main contribution is to provide a lower bound to the $N$-dimensional condenser $p$-capacity of the segment which brings into light its relationship with the $N$-dimensional capacity of a point and more significantly with the $(N-1)$-dimensional capacity of a point. Our lower bound allows to establish the positivity rule for condenser $p$-capacity of a segment. This new method can be extended to study the $p$-capacity of a plane rectangle and by induction for closed boxes in higher dimensions.

Introducing elliptical condensers, we provide an estimate for the condenser 2-capacity of a segment in the plane. The asymptotic expansion follows when the length of the segment goes down to zero. Comparing with results obtained for spherical condensers, it turns out that the topological gradient of the 2-capacity is not an appropriate tool to separate curves and obstacles with non-empty interior in 2D. One way out could be to consider different values of parameter $p$. Another way might be to sort out shapes of obstacles according to the second order of the topological asymptotic expansion, that is considering the topological hessian.

When $p \neq 2$, elliptical condensers may prove useful to further obtain estimates of condenser $p$ capacities of segments.

In the wake of Hausdorff's definition of non-integer dimensions, the question of the $p$-capacity of a segment offers a practical study case of a differential operator operating in between two dimensions, related to two orthogonal directions.

On the basis of this article, research about estimates and asymptotic expansions of condenser $p$-capacities of obstacles with empty interior, may now develop in several directions:

- the study of the convergence speed of descending continuity, in the case the limit compact is a segment or more generally any compact with empty interior;

- the search for more powerful tools resorting to the field of measure transportation, which could be able to grasp the anisotropy caused by the segment;

- the development of methods based on elliptical condensers to obtain further estimations of capacities of segments; 
- the ability of the asymptotic expansion of a $p$-capacity, at first or second order, to sort out curves and obstacles with non-empty interior in $2 \mathrm{D}$ and in $3 D$;

- the study of similar questions for other obstacles in higher dimensions, such as surfaces in $\mathbb{R}^{4}$.

\section{REFERENCES}

[1] S. Amstutz, I. Horchani, M. Masmoudi, Crack detection by the topological gradient method, Control Cybernet., 34(1):81-101, 2005.

[2] S. Amstutz, Topological sensitivity analysis for some nonlinear PDE systems, J. Math. Pures Appl. 85, 540 557, 2006.

[3] S. Amstutz, A.A. Novotny, N. Van Goethem, Minimal partitions and image classification using a gradient-free perimeter approximation, http://hal.archives-ouvertes.fr/hal-00690011, 2012.

[4] H. Attouch, G. Buttazzo, G. Michaille, Variational analysis in Sobolev and BV Spaces, MPS-SIAM Series on Optimization, 2006.

[5] D. Auroux, From restoration by topological gradient to medical image segmentation via an asymptotic expansion, Math. Comput. Model., 2009.

[6] D. Auroux, M. Masmoudi, Image processing by topological asymptotic analysis, ESAIM, Proc. Mathematical methods for imaging and inverse problems, 26:24-44, 2009.

[7] L. J. Belaid, M. Jaoua, M. Masmoudi, L. Siala, Image restoration and edge detection by topological asymptotic expansion, C. R. Math. Acad. Sci. Paris, 342(5):313-318, 2006.

[8] L. J. Belaid, M. Jaoua, M. Masmoudi, L. Siala, Application of the topological gradient to image restoration and edge detection, Engineering Analysis with Boundary Elements, 32(11):891-899, 2008.

[9] J.E. Brothers, W.P. Ziemer, Minimal rearrangements of Sobolev functions, Acta Universitatis Carolinae. Mathematica et Physica, Vol. 28, No. 2, 13-24, Karolinum, Publishing House of Charles University, Prague, 1987.

[10] A. Burchard, A short course on rearrangement inequalities, Copyright (C) Almut Burchard, http://www.math.toronto.edu/almut/, 25 June 2009.

[11] G. Choquet, Theory of capacities, Annales de l'Institut Fourier, tome 5, 113-140, 1954.

[12] A. Ferone, R. Volpicelli, Minimal rearrangements of Sobolev functions: a new proof, Ann. I. H. Poincaré - AN 20,2, 333-339, 2003.

[13] I. Fragalà, F. Gazzola, M. Pierre, On an isoperimetric inequality for capacity conjectured by Pólya-Szegö, Journal of Differential Equations, Volume 250, Issue 3, Pages 1500 -1520, 2011.

[14] D. Gilbarg, N.S. Trudinger, Elliptic Partial Differential Equations of Second Order, Springer-Verlag, 1999, reprint 2001.

[15] Ph. Guillaume, K. Sid Idris, The topological asymptotic expansion for the Dirichlet problem, SIAM J. Control Optim, Vol 41(4): pp. 1042-1072, 2002.

[16] J. Heinonen, T. Kilpeläinen, O. Martio, Non linear Potential Theory of Degenerate Elliptic Equations, second edition, Dover Publications, 2006.

[17] S. Kichenassamy, L. Véron, Singular solutions of the p-Laplace equation, Mathematishe Annalen, 275, 599-615, 1986.

[18] S. Larnier, J. Fehrenbach, Edge detection and image restoration with anisotropic topological gradient. Acoustics Speech and Signal Processing (ICASSP), 2010 IEEE International Conference, pages 1362 -1365, March 2010.

[19] S. Larnier, J. Fehrenbach, M. Masmoudi, The topological gradient method: From optimal design to image processing, Milan Journal of Mathematics, vol. 80, issue 2, pp. 411-441, December 2012.

[20] P. Lindqvist, Notes on the p-Laplace equation, Summer School in Jyväskylä, 2005.

[21] J. Malý, W.P. Ziemer, Fine regularity of solutions of elliptic partial differential equations, Mathematical Surveys and Monographs, volume 51, American Mathematical Society, 1997.

[22] M. Masmoudi, The topological asymptotic, Computational methods for control applications, Ed. H. Kawarada and J. Périaux, International Series, Gakuto 2002.

[23] V.G. Maz'ya, S. Nazarov and B. Plamenevskij, Asymptotic theory of elliptic boundary value problems in singularly Perturbed Domains, Operator theory, advances and applications, Volumes 1 \& 2, Birkhäuser, 2000.

[24] S.A. Nazarov, J. Sokolowski, Self-adjoint Extensions for the Neumann Laplacian and Applications, Acta Mathematica Sinica, Volume 22, Issue 3, pp 879 -906, May 2006.

[25] G. Pólya, G. Szegö, Inequalities for the capacity of a condenser, American J. Math 67, 1 -32, 1945.

[26] G. Pólya, G. Szegö, Isoperimetric inequalities in mathematical physics, Princeton University Press, 1951.

[27] A. Porretta, L. Véron, Separable p-harmonic functions in a cone and related quasilinear equations on manifolds, arXiv:0710.2974v1 [math.AP], 2007.

[28] B. Samet, S. Amstutz, M. Masmoudi, The Topological Asymptotic for the Helmholtz Equation, SIAM J. Control Optim., 42(5), $1523-1544,2003$.

[29] A. Schumacher, Topologieoptimierung von Bauteilstrukturen unter Verwendung von Lochpositionierungkriterien, Ph.D. Thesis, Universität-Gesamthochschule-Siegen, Siegen, 1995.

[30] J. Serrin, Local behavior of solutions of quasi-linear equations, Acta Math. 113, 247-302, 1964.

[31] J. Serrin, Singularities of solutions of nonlinear equations, Proc. Symp. App. Math 17, 68-88, 1965.

[32] J. Sokolowski, A. Zochowki, On the topological derivative in shape optimization, Technical report \# 3170, INRIA, 1997.

[33] G. Tee, Surface area and capacity of ellipsoids in $n$ dimensions, New Zealand J. Math. 34, 165 -198, 2005. 
[34] P. Tolksdorf, On the Dirichlet problem for quasilinear equations in domains with conical boundary points, Comm. Partial Differential Equations 8, 773-817, 1983.

[35] P. Tolksdorf, Regularity for a more general class of quasilinear elliptic equations, Journal of differential equations 51, 126-150, 1984 .

[36] V.S. Vladimirov, Methods of the Theory of Generalized Functions, Analytical Methods and Special Functions, Taylor \& Francis Ltd, 2002.

[37] L. Véron, Singular p-harmonic functions and related quasilinear equations on manifolds, Luminy conference on Quasilinear Ellipctic and Parabolic Equations and Systems, Electronic Journal of Differential Equations, Conferences 08, 133-154, 2002.

[38] L. Wang, Compactness methods for certain degenerate elliptic equations, Journal of differential equations, 107, 341-350, 1994

[39] E. Weisstein, Elliptic Cylindrical Coordinates, From MathWorld-A Wolfram Web Resource.

[40] Wikipedia, Elliptic coordinate system, http://en.wikipedia.org/wiki/Elliptic coordinate system, last modified on 25 February 2013.

Institut de mathématiques de Toulouse, INSA de Toulouse, Département Mathématiques et Modélisation, 135, Avenue de Rangueil, 31077 Toulouse, France.

E-mail address: alain.bonnafe@insa-toulouse.fr 